\title{
¿Cuál es la Cantidad Transfinita Posible de dígi- tos Posteriores al Punto Decimal que Confor- MAN LA EXPANSIÓN DE UN NÚMERO TRASCENDENTE?
}

\author{
ALEJANDRO MATUS
}

\begin{abstract}
RESUMEN. Se prueba que, si se asume como consistente el proceso diagonal de Cantor, es también lógicamente consistente y necesario deducir que entre los números trascendentes existen diferentes cardinalidades transfinitas en la cantidad de los dígitos que los componen en su expansión después del punto decimal: Algún trascendente puede estar formado por una cantidad $\aleph_{0}$ de cifras después del punto, mientras que otro por $\aleph_{1}$ o $\aleph_{2} \mathrm{o}$ $\aleph_{\alpha}$ dígitos. Entonces, procedemos a construir matemáticamente tales cardinalidades.

Ello obliga a deducir también la existencia, dentro de la clase $\mathbb{R}$ de los números reales, de infinitos subconjuntos $\mathrm{R}_{\alpha}$ de tales números, con $\aleph_{\alpha}$ cardinalidades diferentes uno de otro, que procedemos a construir; y a partir de ello se encuentra la inadmisibilidad lógica de identificar, como inadvertidamente en la teoría actual, a $\mathbb{R}$ mismo con el que en realidad es un subconjunto $\mathrm{R}_{1}$ estrictamente propio de $\mathbb{R}$.

Obteniendo para $\mathbb{R}$ un nuevo teorema, análogo al Teorema de Cantor sobre los conjuntos potencia. Excepto que este nuevo teorema nos lleva a redimensionar las cardinalidades actualmente aceptadas de los conjuntos potencia $\mathrm{P}_{\alpha}$. Estableciendo que ninguno de ellos alcanza la cantidad de elementos de $\mathbb{R}$. Y para toda $\alpha$ la cardinalidad de cada $\mathrm{P}_{\alpha}$ es igual a la cardinalidad de cada $\mathrm{R}_{\alpha}$; es decir, en ambos casos, a $\aleph_{\alpha}$.

Así, los números reales $r \in \mathbb{R}^{\prime}$ tienen una riqueza infinitamente mayor de la que se ha conceptualizado hasta ahora en la Teoría de Conjuntos y el Análisis Matemático.

También es demostrado que, suponiendo que el método diagonal de Cantor sea consistente, es necesario reformular la Hipótesis del Continuo.
\end{abstract}

Palabras clave: NÚMEROS REALES_NÚMEROS TRASCENDENTES_INFINITO_ANÁLISIS MATEMÁTICO_CONJUNTOS POTENCIA_CARDINALIDAD_DIAGONAL_CANTOR_HIPÓTESIS DEL CONTINUO _IRRACIONALES

INTRODUCCIÓN. UNA REFORMULACIÓN DE LA INFINITA RIQUEZA DEL CUERPO DE LOS NÚMEROS REALES, LOS CONJUNTOS POTENCIA Y LA HIPÓTESIS DEL CONTINUO

A partir del resultado del método de la diagonal de Cantor, con la existencia de transfinitas cantidades $\aleph_{\alpha}$, es investigado: ¿cuántas cifras decimales componen a un número trascendente? $i \aleph_{0}, \aleph_{1}$, una cantidad infinita potencial? Y se encuentra que, utilizando las consecuencias estrictamente lógicas de tal método, necesariamente se deduce que entre los números trascendentales existen distintas cantidades transfinitas de dígitos en su construcción.

Esto es, existen números trascendentes elementos de $\mathbb{R}$ con $\aleph_{0}$ dígitos en su desarrollo después del punto decimal, pero hay también trascendentales con $\aleph_{1}$ o $\aleph_{2}$, o $\aleph_{\alpha}$ cifras. Cardinalidades en la cantidad de cifras de tales elementos que procedemos a construir matemáticamente. Desde aquí deducimos, extendiendo en una forma justificada lógicamente el panorama del procedimiento diagonal, la inesperada conclusión de que la clase $\mathbb{R}$ de los números reales está formada por un número infinito de $\mathrm{R}_{\alpha}$ subconjuntos que difieren en sus cardinalidades unos de otros. En otras palabras, que existen subconjuntos propios de $\mathbb{R}$ con $\aleph_{0}$ o $\aleph_{1}$ cantidad de elementos, pero también otros con $\aleph_{2}, \aleph_{100}, \aleph_{\omega}$ o 
cualquier $\aleph_{\alpha}$ cantidad de números reales como miembros. Cardinalidades en la cantidad de elementos de tales subconjuntos que también procedemos a construir.

Así, los números reales tienen una mayor riqueza de la que hasta ahora ha sido conceptualizada en la Teoría de Conjuntos y el Análisis Matemático (y en Teoría de la Medida, Topología, etc.).

Entonces, si asumimos como consistentes el método de la diagonal de Cantor y las pruebas elaboradas por la Teoría de Conjuntos respecto a la función $\mathrm{f}: \mathbb{N} \rightarrow \mathbb{R} i i$ (con $\mathbb{N}$ como el conjunto de los números naturales y $\mathbb{R}$ ii el conjunto de los números reales en el intervalo $[0,1))$, encontramos la improcedencia lógica de identificar, como se hace en la teoría vigente, a $\mathbb{R}$ mismo con un subconjunto $R_{1}$, estrictamente propio de $\mathbb{R}$.

Arribamos así a un teorema referente a los números reales que es análogo al Teorema de Cantor sobre los conjuntos potencia $\mathrm{P}_{\alpha}$. Excepto que este nuevo teorema además nos lleva a redimensionar las cardinalidades actualmente aceptadas de los conjuntos potencia. Estableciendo que ninguno de ellos alcanza a la cantidad de elementos de $\mathbb{R}$. Y para toda $\alpha,\left|P_{\alpha}\right|=\left|R_{\alpha}\right|=\aleph_{\alpha}$.

Fundados en estas consideraciones, también es probado que la Hipótesis del Continuo, tal como está formulada en la actualidad, i.e. a partir de $2^{\mathrm{Na}_{\alpha}}=\mathrm{c}$, es falsa (siendo c la cardinalidad del continuo). De acuerdo con eso, se demuestra que entre $\mathbb{N}$ y el continuo existen infinitos conjuntos $\mathrm{R}_{\alpha}$ con $\aleph_{\alpha}$ cardinalidades diferentes $\mathrm{y}$, para todo $\alpha, 2^{\mathrm{N} \alpha}<\mathrm{c}$.

Sin embargo surge, y planteamos, un problema parecido, pero que ya no se refiere al continuo como un todo, que llamamos Hipótesis de la Continuidad Dentro del Continuo. Y espera por una solución la cuestión de si entre estos $\mathrm{R}_{\alpha}$ (o sus $\mathrm{P}_{\alpha}$ equivalentes) existen otros conjuntos intermedios con cardinalidades distintas a las de aquellos $\aleph_{\alpha}$ obtenidos por el proceso diagonal.

Para hacer eso se parte en la sección 1 del método de la diagonal de Cantor, con la conocida proposición de la Teoría de Conjuntos que afirma que el conjunto de los números reales no es enumerable. Asimismo se establecen en el apartado 1.1 algunas definiciones basadas en el análisis del proceso de la diagonal que nos auxiliarán en nuestra argumentación.

En el punto 2, encontramos que la demostración por la diagonal de Cantor tiene la limitación lógica estricta de únicamente permitir concluir que en la función $\mathrm{f}: \mathbb{N} \rightarrow \mathbb{R} i i$, el proceso diagonal sobre $\mathbb{R} i i$ genera un número real $\mathrm{r}_{\mathrm{i}}$ formado por una cantidad $\boldsymbol{N}_{0}$ de cifras. Y a partir de ese número sólo se puede generar un número antidiagonal con la misma cantidad $\aleph_{0}$ de dígitos. Esta constatación es usada como premisa para demostrar que existe un subconjunto de $\mathbb{R} i i$ cuyos elementos a lo más están formados por una cantidad $\aleph_{0}$ de dígitos (subconjunto al cual llamamos $R \uparrow_{\aleph_{0}}$ o $R R_{1}$. Y tal que $\left|R \Gamma_{\aleph_{0}}\right|=\aleph_{1}$ ) (el subíndice $\aleph_{0}$ indica la restricción acerca de la cantidad máxima de cifras y el subíndice 1 indica que es el primer $\aleph$ obtenido por la diagonal después de $\aleph_{0}$, sin presuponer que entre $\aleph_{0}$ y $\aleph_{1}$ pueda existir o no un $\aleph$ intermedio); y $|\mathbb{R} i|>\left|R \aleph_{\aleph_{0}}\right|>|\mathbb{N}|$.

Por lo tanto, para que $\aleph_{1}$ fuera igual a la cantidad de elementos de $\mathbb{R}$ ii, i.e. a la cantidad de elementos de $\mathbb{R}$, sería necesario que la cantidad máxima de dígitos que componen a cualquier número real fuera $\aleph_{0}$.

Para determinar si ésto es así o no, comenzamos en el punto 3.1 con la demostración de que, si establecemos la función $\mathrm{f}: \mathrm{R} \boldsymbol{\Gamma}_{\boldsymbol{N}_{0}} \rightarrow \mathbb{R} \mathbf{i}$, podemos aplicar en $\mathbb{R}$ ii la que llamamos 1 ampliación de la diagonal de Cantor, que demuestra que existe otro subconjunto de $\mathbb{R} i i$ mayor que $\left|R_{1}\right|=\aleph_{1}$, al que nombramos $R_{2}$. El cual, como se prueba en el apartado 3.2, tiene elementos, números reales, conformados a lo más por $\aleph_{1}$ cifras, y por ello también lo llamamos $\mathrm{R} \uparrow_{\mathrm{N}_{1}}$. 
Todo esto nos obliga a redimensionar al conjunto potencia $\mathrm{P}(\mathbb{N})$ en el inciso 3.3 , obteniendo los resultados de que $\left|\mathrm{R}_{1}\right|=|\mathrm{P}(\mathbb{N})|,\left|\mathrm{R}_{1}\right|=2^{\mathrm{N}_{0}}$, y $\mid \mathbb{R}$ i $i|>| \mathrm{P}(\mathbb{N}) \mid$.

Por otra parte, para que ocurriese que la cantidad de elementos de $\mathbb{R}$ ii fuera igual a la de $\mathrm{R}_{2}$, o sea a $\aleph_{2}$, sería necesario que la cantidad máxima posible de cifras de un número real fuera $\aleph_{1}$.

Más en general: ¿Es igual $\mathbb{R} i i$ a algún $\mathrm{R} \uparrow_{\aleph_{\alpha}}$, cuyos elementos tengan una cantidad máxima de $\aleph_{\alpha}$ dígitos? ¿Acaso no existen los cardinales $\left|R_{\alpha}\right|=\aleph_{\alpha}$ y $\left|R_{\alpha+1}\right|=\aleph_{\alpha+1}$. Y por lo tanto, acaso no existen siempre números reales con más que cualquier cantidad dada de $\aleph_{\alpha}$ cifras?

Para saberlo, en el apartado 4 se generaliza el argumento de la diagonal de Cantor, aplicado sobre los subconjuntos transfinitos de $\mathbb{R} i i$, demostrando iterativamente el caso en el cual $\alpha$ implica $\alpha+1$.

Con tal fin argüimos, aplicando la que nombramos $\delta$-ampliación de la diagonal de Cantor, que si suponemos la existencia de cualquier conjunto de números reales $\mathrm{R}_{\alpha}\left(\equiv \mathrm{R} \uparrow_{\aleph_{\alpha-1}}\right)$, subconjunto de $\mathbb{R}$ ii, cuyos elementos poseen una cantidad de cifras restringida a un máximo de $\aleph_{\alpha-1}$, entonces necesariamente existe un conjunto $R_{\alpha+1}$ sucesor suyo cuya cardinalidad es mayor que la de $\mathrm{R}_{\alpha}$. $\mathrm{Y}$ los elementos de $\mathrm{R}_{\alpha+1}$ son cada uno un número real con una cantidad a lo máximo de $\aleph_{\alpha}$ cifras en su composición. Con esto hemos verificado y construido de manera matemática las cantidades permitidas de dígitos en cada número real: finitas, $\aleph_{0}, \aleph_{1}, \ldots, \aleph_{\alpha}$.

Con base en ello, se deduce que, para todo $\alpha, R_{\alpha} \subset \mathbb{R} i$ y $|\mathbb{R} i|>\left|R_{\alpha}\right|$. Es decir, no existe un último número transfinito $|\mathrm{R}|(=\aleph)$, pues a cada número $|\mathrm{R}|$ le sigue otro número $|\mathrm{R}|$ mayor. No existe un último conjunto $\mathrm{R}_{\alpha}=\mathbb{R} i i$ con una cardinalidad última. Por lo tanto, en la colección de los subconjuntos $\mathrm{R}_{\alpha}$ de la clase $\mathbb{R}$ ii se cumple la propiedad de orden arquimediano.Y cualquier número $r_{\alpha} \in \mathrm{R}_{\alpha}$ tiene que ser un número real. Existiendo siempre números reales con más de cualquier $\aleph_{\alpha}$ cantidad de cifras.

Extendiendo lo ya obtenido, se procede en el apartado 5 a la consecuente renormalización de las clases $\mathrm{R}_{\alpha}, \mathrm{P}_{\alpha}$, $\mathbb{R}$ ii $\mathrm{y}, \mathbb{R}$. Con $\mathrm{P}_{\alpha} \equiv \alpha$-ésimo conjunto potencia a partir de $\mathbb{N}$. Y obtenemos los teoremas $\left|\mathrm{R}_{\alpha}\right|=\left|\mathrm{P}_{\alpha}\right| \mathrm{y}|\mathbb{R} i|>\left|\mathrm{P}_{\alpha}\right|$.

Por el error de identificar a $R_{1}$ con $\mathbb{R} i \dot{i}$ o con $\mathbb{R}$ también se piensa que a partir de $\aleph_{2}$, o cualquier cardinal transfinito que corresponda al conjunto potencia $\mathrm{P}(\mathrm{P}(\mathbb{N})) \equiv \mathrm{P}_{2}$, se rebasa al conjunto $\mathbb{R}$ de todos los números reales. Cuando $\left|\mathrm{P}_{2}\right|$ a lo que rebasa es a $\left|\mathrm{R}_{1}\right|$, pero es igual a $\left|\mathrm{R}_{2}\right|$. Y $\left|\mathrm{P}_{\alpha}\right|$ a lo que rebasa es a $\left|\mathrm{R}_{\alpha-1}\right|$, y es igual a $\left|\mathrm{R}_{\alpha}\right|$ y menor a $|\mathbb{R} i i|$. En realidad no se ha ido más allá de $\mathbb{R}$ ¿i ni de $\mathbb{R}$. Cualquier número $\mathrm{r}_{\alpha} \in \mathrm{R}_{\alpha}$ tiene que ser un número real $r_{i} \in \mathbb{R} i$ i.

En el inciso 6 se hace explícito cómo de los teoremas demostrados se deduce que, si se asume que el método diagonal de Cantor es consistente, entonces la Hipótesis del Continuo (HC), como es concebida en la actualidad, es falsa. Es falso que si $\mathrm{R}_{\alpha} \subset \mathbb{R}$ es un conjunto no enumerable por $\mathbb{N}$, entonces existe una biyección $f: R_{\alpha} \rightarrow \mathbb{R}$. O sea, no es verdadero que $R_{\alpha} \subset \mathbb{R} \wedge \aleph_{\alpha}>\aleph_{0} \leftrightarrow \aleph_{\alpha}=|\mathbb{R}|$.

Lo importante es que esto quiere decir que entre $\mathbb{N}$ y el continuo $\mathfrak{c}=|\mathbb{R}|$ hay una infinita cantidad de conjuntos infinitos determinados, $\mathrm{R}_{\alpha}$, con cardinalidades distintas entre sí. Cada uno de ellos formado por números reales. $Y$, además $2^{\aleph_{0}}$ no es la cardinalidad del continuo, puesto que $c>2^{\aleph_{0}}$. Siendo $2^{\aleph_{0}}=\aleph_{1}=\left|R_{1}\right|$.

Esta refutación de la existente Hipótesis del Continuo tiene la ventaja de permitirnos ver rigurosamente el alcance del cuerpo de los números reales, frente a meras conjeturas intuitivas respecto a la riqueza del mismo continuo. 
Pero en el rubro 7 planteamos una reformulación, al advertir dos nuevas Hipótesis, a las que llamamos Hipótesis de la Continuidad Interior al Continuo (HCIC):

1. La Hipótesis Restringida: $\mathrm{Si} \mathrm{R}_{\beta} \subset \mathrm{R}_{1}$ es un conjunto no enumerable por $\mathbb{N}$, entonces existe una biyección $\mathrm{f}: \mathrm{R}_{\beta} \rightarrow \mathrm{R}_{1}$.

2. La Hipótesis Generalizada: $\mathrm{Si} \mathrm{R}_{\beta} \subset \mathrm{R}_{\alpha+1}$ es un conjunto no $\aleph_{\alpha-\text { enumerable por }} \mathrm{R}_{\alpha}$, entonces existe una biyección $\mathrm{f}: \mathrm{R}_{\beta} \rightarrow \mathrm{R}_{\alpha+1}$. $\mathrm{Y}$ así tenemos $\mathrm{R}_{\beta} \subset \mathrm{R}_{\alpha+1} \wedge\left|\mathrm{R}_{\beta}\right|>\left|\mathrm{R}_{\alpha}\right|$ $\leftrightarrow\left|R_{\beta}\right|=\left|R_{\alpha+1}\right|$. Esto es, para cualquier operación matemática posible, además de ser válido para la diagonal de Cantor:

$\forall \alpha \aleph_{\alpha+1}=2^{\aleph_{\alpha}}=2^{\mid R_{\alpha}}|=2| P_{\alpha}|=| P_{\alpha+1}|=| R_{\alpha+1} \mid(\neq|\mathbb{R}|)$.

En este replanteamiento, con ambas presentaciones de la HCIC de ninguna manera pueden, $\aleph_{\alpha+1}$ ó $2^{\aleph_{\alpha}}, \aleph_{\alpha}$-enumerar a todos los reales $\mathbb{R}$. Pues ambas solamente $\aleph_{\alpha}$-enumeran a $\mathrm{R}_{\alpha+1} \subset \mathbb{R}$. La pregunta sigue siendo si entre $\aleph_{\alpha} \mathrm{y} 2^{\mathrm{N}_{\alpha}}$ (ambos dentro del continuo y menores en cardinalidad a él) existe otro cardinal transfinito. Pero ya no es equivalente a si entre un $\aleph_{\alpha}$ interior al continuo y el continuo $\mathfrak{c}$ existe un cardinal intermedio. Puesto que ningún $2^{\aleph_{a}}$ es la cardinalidad del continuo.

Con todo ello, si asumimos que la $\mathrm{HC}(\neq \mathrm{HCIC})$ es verdadera, entonces el método de la diagonal de Cantor es inconsistente. Pero lo que afecta a la HC no afecta a la HCIC. Respecto a ésta, y a su negación, sí falta probar si es verdadera o falsa, o consistente o inconsistente en relación al método de la diagonal o bien a diferentes axiomas.

Finalmente, la diferencia importante con el Teorema de Cantor es que la demostración aquí presentada es realizada directamente con la generalización de la aplicación del método diagonal sobre el conjunto $\mathbb{R} i i$ de los números reales. Pero solamente así logramos ver algo que no permite apreciar el Teorema de Cantor al hacer referencia a los conjuntos potencia: El calado de los números reales y su real alcance respecto a los conjuntos transfinitos.

\section{PUNTOS DE PARTIDA}

Entonces, en este escrito son tomadas como base de inicio las premisas y el método utilizados en el proceso diagonal de Cantor, tal como son permitidos por ZFC y axiomáticas similares (cf. por ejemplo en Jech [13], Fraenkel [8] o Gödel [11]), que conducen a la deducción de la existencia de distintas cardinalidades entre conjuntos infinitos. Y avanzando desde ahí obtenemos nuevos teoremas respecto a los números reales y a los conjuntos y números transfinitos.

Se parte, así, de la demostración constructiva y directa del teorema (cf. Fraenkel [8], pp. 54, 57 y 58; Fraenkel [9], pp. 36 y 37; y Gray [12], p. 823), la cual establece que el conjunto de los números reales no es enumerable: Como se sabe, en la función $\mathrm{f}: \mathbb{N} \rightarrow$ $\mathbb{R} i i$ (donde los $n \in \mathbb{N}$ números naturales del dominio y los $r_{i} \in \mathbb{R} i$ números reales en el contradominio, en el intervalo $[0,1) \in \mathbb{R}$, son dispuestos en correspondencia), a cada dígito de cada real $r_{i} \in \mathbb{R} i i$, en su parte decimal, se le puede representar como apq, donde $p$ indica el lugar que ocupa en la enumeración el $r_{i}$ al que pertenece la cifra respectiva; $y$ q el lugar que ocupa la misma cifra dentro de tal $r_{i}$.

De aquí se deriva que podamos efectuar un arreglo como en la Tabla 1, donde p y q corresponden respectivamente al renglón y la columna en que tal dígito está ubicado.

Luego, sobre el conjunto $\mathbb{R}$ ii es operada una función diagonal $f_{d}=\{(p, q) \in A \times A: p=q\}$ que selecciona de cada $r_{i}$ a una cifra tal que $p=q$, con lo cual se forma un número real diagonal $0 . a_{11}$ a 22 a 33 a $44 \ldots$. 
Operando con este real se genera un número real antidiagonal $r_{a} \in[0,1)$. Por ejemplo, estableciendo una función característica que sustituye sobre el real diagonal a cualquier dígito que difiera de 1 por $1 \mathrm{y}$ a toda cifra igual a 1 por 2 . Antidiagonal $r_{a}$ que difiere de cualquiera de los números en el rango $\mathbb{R} i i$, es decir, que no pertenece a este rango inyectado desde el dominio $\mathbb{N}$. Demostrando con eso que si bien hay inyección, no hay suprayección y por lo tanto que $\mathbb{R} i i$ es un infinito con cardinalidad mayor que $\mathbb{N}$.

\begin{tabular}{|l|l|}
\hline $\mathbb{N}$ & $\mathbb{R i}$ \\
\hline 1 & $0 . \mathbf{a} 11$ a12 a13 a14 a15 $\ldots$ \\
\hline 2 & 0. a21 a22 a23 a24 a25 $\ldots$ \\
\hline 3 & 0. a 31 a32 a33 a34 a35 $\ldots$ \\
\hline 4 & 0. a41 a42 a43 a44 a45 $\ldots$ \\
\hline$\ldots$ & $\ldots$ \\
\hline
\end{tabular}

TABLA 1. f: $\mathbb{N} \rightarrow \mathbb{R} i i$

\subsection{Definiciones.}

Presuponemos, recalcamos, las características y la consistencia del procedimiento de la diagonal de Cantor. Examinando este proceso, cabe destacar la diferencia entre lo que llamaremos un elemento diagonal cognado (emparentado) y un elemento diagonal ajeno.

Definición 1. Diagonal cognado. Es el elemento $e_{c}$ en una función f: dominio $\mathrm{D} \rightarrow$ contradominio $\mathrm{C}$, obtenido por el proceso de la diagonal de Cantor sobre $\mathrm{C}$, cuando también es necesario concluir que (por definición de los elementos de C) e e $\in$ C. Así, por ejemplo, el número diagonal de Cantor $\mathrm{r}_{\mathrm{c}}$ sobre $\mathbb{R}$ ii es un diagonal cognado, al ocurrir también que, por definición de número real, $r_{c} \in \mathbb{R} i i$.

Definición 2. Antidiagonal cognado. Es el elemento $\neg e_{c}$ obtenido de una función $\mathrm{f}\left(\mathrm{e}_{\mathrm{c}}\right)=$ $\neg e_{c}$ aplicada a las cifras que componen a un elemento $e_{c}$ diagonal cognado. Y también es necesario que, por definición de los elementos de $\mathrm{C}, \neg \mathrm{e}_{\mathrm{c}} \in \mathrm{C}$.

Definición 3. Diagonal ajeno. El elemento diagonal no cognado: cuando a e no le toca pertenecer necesariamente a $\mathrm{C}$.

Definición 4. Antidiagonal ajeno. El elemento antidiagonal no cognado.

Por otra parte, a partir de que es llamado enumerable todo conjunto que tenga una correspondencia biunívoca con $\mathbb{N}$, generalizamos esa definición para referirnos a las distintas comparaciones de tipo cantoriano entre conjuntos infinitos de cardinalidades diferentes que aquí haremos:

Definición 5. $\aleph_{\alpha}$-enumerar ( $\forall \alpha$ número ordinal), es relacionar la cardinalidad de un conjunto infinito cualquiera $\mathrm{A}$ con la de otro $\mathrm{B}$, en una función $\mathrm{f}: \mathrm{A} \rightarrow \mathrm{B}$. Con lo cual se determina si existe correspondencia biunívoca entre ellos.

Por ejemplo, todo conjunto infinito en correspondencia biunívoca con $\mathbb{N}$ diremos es $\aleph_{0-}$ enumerable; todo conjunto infinito en correspondencia biunívoca con $\mathrm{P}(\mathbb{N})$, el conjunto de los subconjuntos de $\mathbb{N}$, es $\aleph_{1}$-enumerable; etc. 
Cuando en $\mathrm{f}: \mathbb{N} \rightarrow \mathbb{R}$ ii Cantor traza el elemento diagonal cognado $0 . \mathrm{a}_{11}$ a22 a33 a44 ... sobre el conjunto $\mathbb{R}$ ii del contradominio y a partir de él genera al número real antidiagonal $\mathrm{r}_{\mathrm{a}} \in$ $[0,1)$, demuestra que no existe alguna $\aleph_{0}$-enumeración de los reales capaz de dar cuenta de todos ellos en el intervalo [0,1): $\mid \mathbb{R}$ ii $\mid$ (la cantidad de elementos de $\mathbb{R}$ ii) $>|\mathbb{N}|$ (la cardinalidad de $\mathbb{N}$ ). Y por lo tanto existen $\aleph_{1}$ y $\aleph_{0}$ tales que $\aleph_{1}>\aleph_{0}$.

Se aclara: Lo llamamos $\aleph_{1}$, más que simplemente $\aleph$, por ser el primer número cardinal generado por el proceso de la diagonal de Cantor desde $\mathrm{f}: \mathbb{N} \rightarrow \mathbb{R} i$. Sin presuponer que a partir de otro procedimiento, o como axioma, puedan o no puedan obtenerse otros $\aleph$ intermedios entre él y $\aleph_{0}$, a los cuales, si es el caso, y con esta convención, se podría llamar, por ejemplo, $\aleph_{1 / 2}$ ó $\aleph_{0.5}$, ó $\aleph_{1 / 3}$, etc.

Además, asumimos que en la Teoría de Conjuntos ya está demostrado que la cantidad de elementos de $\mathbb{R}$ ii es igual a la cantidad de elementos de $\mathbb{R}$. Y que $\aleph_{1}=|\mathrm{P}(\mathbb{N})|>|\mathbb{N}|$.

2. NOVEDADES: UNA DEDUCCIÓN LÓGICAMENTE NECESARIA A PARTIR DEL ARGUMENTO DE LA DIAGONAL, ACERCA DE LA MÁXIMA CANTIDAD DE CIFRAS POSIBLE EN CADA NÚMERO REAL $r_{i} \in \mathbb{R}$ ii.

A partir de los supuestos y deducciones del proceso diagonal de Cantor queremos destacar conclusiones que son lógicamente necesarias, pero no observadas hasta la actualidad en la Teoría de Conjuntos ni en el Análisis Matemático (ni en Topología, etc.).

En primer lugar, con lógica estricta, la demostración por la diagonal de Cantor sobre los números reales tiene la limitación de únicamente permitir concluir algo que parece simple, pero tiene fuertes consecuencias, como lo probaremos más adelante:

Teorema 1. En la función $f: \mathbb{N} \rightarrow \mathbb{R}$ ii, el proceso diagonal sobre $\mathbb{R} \ll i$ construye un número real trascendente $\mathrm{r}_{\mathrm{i}}$ formado por una cantidad $\boldsymbol{N}_{0}$ de cifras. Y a partir de ese número diagonal se construye otro número antidiagonal trascendente con la misma cantidad $\aleph_{0}$ de dígitos.

Demostración: En la función $\mathrm{f}: \mathbb{N} \rightarrow \mathbb{R} i$, al inyectar el dominio $\mathbb{N}$ en $\mathbb{R}$ ii, es en un subconjunto (sea propio o igual) de éste contradominio $\mathbb{R}$ ii que se aplica la función diagonal, la cual construye un número real diagonal, que es cognado (emparentado) por ser él mismo un número real.

Es decir, tal subconjunto en el contradominio contiene necesariamente una cantidad $\aleph_{0}$ de elementos, por estar cada uno de ellos enumerado por algún único número natural, por planteamiento de inicio.

Y con base en ello se obtiene el elemento antidiagonal que demuestra que $|\mathbb{R} i|>|\mathbb{N}|$, haciendo diferir, con la función $\mathrm{f}\left(\mathrm{e}_{\mathrm{c}}\right)=\neg \mathrm{e}_{\mathrm{c}}$, una a una cada cifra ars del antidiagonal cognado respecto a la correspondiente cifra apq del diagonal cognado.

O sea, cualquier número real al que represente el elemento diagonal cognado obtenido sobre $\mathbb{R} i i$ (y por ende cualquier número real correspondiente al antidiagonal cognado de ella derivado con un cambio uno a uno de sus cifras) sólo puede tener en su composición una cantidad de dígitos igual a la cardinalidad de los números naturales $\left(\aleph_{0}\right)$, en virtud de la forma de construirlo.

Y la función diagonal es operada, por hipótesis de inicio, sobre un rango (en el contradominio $\mathbb{R}$ ii) al que pertenece una cantidad de números reales igual a la cantidad de los números naturales que los $\aleph_{0}$-enumeran uno a uno desde el dominio $\mathbb{N}$.

La diagonal no puede ser aplicada, en la prueba de Cantor, sobre un conjunto de los números reales en un rango que rebase la cantidad de $\aleph_{0}$ (los $\aleph_{0}$ renglones), pues en esa 
demostración sería una petición de principio presuponer que ya existe, antes de operar la diagonal, al menos un número real $0 . a_{n+x} 1 a_{n+x} 2 a n+x 3 a n+x 4 \ldots a n+x$ ri que rebasa al rango de $\mathbb{R}$ ii en la sucesión; es decir presuponer que el contradominio es mayor que el conjunto de imágenes de $\mathbb{N}$. Que es tanto como presuponer que no existe suprayección antes de demostrar que la suprayección no existe.

Entonces, subrayo, ese elemento diagonal cognado es obtenido, por planteamiento de inicio, sobre un rango en el contradominio $\mathbb{R} i i$ al que pertenece una cantidad de números reales igual a la cantidad de los números naturales que los inyectan uno a uno desde la otra columna (el dominio $\mathbb{N}$ ).

Preparando la prueba del Teorema 3 , de que existe un conjunto $R \Gamma_{N_{0}}$ tal que $\mid \mathbb{R}$ i $\mid>$ $\left|\mathrm{R}_{\boldsymbol{N}_{0}}\right|>|\mathbb{N}|$, se demuestran los lemas, el Teorema y los corolarios siguientes.

Lema 1. Sea $C r_{i} \equiv$ Cantidad de cifras que integran a cada número real $r_{i} \in \mathbb{R} i i$.

Existe un conjunto $R \uparrow_{\aleph_{0}}$ tal que sus elementos $r_{\mathrm{i}}$ son cada uno un número real que consta a lo máximo de una cantidad de $\aleph_{0}$ cifras, y puede ser estrictamente igual a $\mathbb{R}$ ¿i o bien a un subconjunto propio del mismo:

$\exists R \uparrow_{\aleph_{0}}=\left\{r_{i} \in \mathbb{R} i|| C r_{i} \mid \leq \aleph_{0}\right\} \subseteq \mathbb{R} i$.

Demostración. A partir del conjunto del rango en $\mathbb{R} i i$ inyectado por el dominio $\mathbb{N}$ (al que llamaremos $\mathbb{R}^{\prime} i_{i N}$ ) se construye un nuevo subconjunto de $\mathbb{R}$ ii , al que se nombrará $R \uparrow_{\text {i }}$ (que, hasta aquí, puede ser subconjunto propio o igual de $\mathbb{R}$ ii; esto es, si se diera este último caso, sería tan solo un nombre distinto de $\mathbb{R}(i)$, con la restricción de que sus elementos $r_{i} \in \mathbb{R} i i$ sólo pueden poseer hasta $\aleph_{0}$ cifras: $\mathrm{Cr}_{\mathrm{i}} \leq \aleph_{0}$ (por el Axioma Esquema de Separación).

Por el Axioma Esquema de Separación formamos también, a partir de $\mathbb{R} i i$, un conjunto integrado por cualquier número real implicado por la antidiagonal sobre $\mathbb{R} i i$.

En el subconjunto $\mathrm{R} \uparrow_{\aleph_{0}}$ unimos los elementos del conjunto $\mathbb{R}^{\text {iin }}$ con los elementos del conjunto formado por cualquier número real implicado por la antidiagonal sobre $\mathbb{R} i i$ (por el Axioma de Unión), mismos que cumplen también (por el Teorema 1) con la restricción estipulada $\mathrm{Cr}_{\mathrm{i}} \leq \aleph_{0}$.

Se ha construido así el nuevo conjunto $\mathrm{R}_{\aleph_{0}}=\left\{\mathrm{r}_{\mathrm{i}} \in \mathbb{R} \boldsymbol{i} i|| \mathrm{Cr}_{\mathrm{i}} \mid \leq \boldsymbol{\aleph}_{0}\right\} \subseteq \mathbb{R}$ ii.

Se recalca, se le asigna a $\mathrm{R}\left\lceil\aleph_{0}\right.$ el subíndice $\aleph_{0}$ para indicar con él la restricción, derivada del procedimiento de la diagonal, acerca de la cantidad máxima de cifras que pueden alcanzar los números reales que pertenecen a tal conjunto. Y para destacar más adelante su correlación con los subíndices de los números transfinitos $\aleph_{\alpha}$, también diré $R \uparrow_{\aleph_{0}} \equiv R R_{1}$.

Teorema 2. La cardinalidad de $R \uparrow_{\aleph_{0}}$ es mayor a la de $\mathbb{N} y$ menor o igual a la de $\mathbb{R}$ Ri:

$|\mathbb{R} i| \geq\left|R_{\aleph_{0}}\right|>|\mathbb{N}|$. Es decir, $|\mathbb{R} i i| \geq\left|R_{1}\right|>\left|R_{0}\right|$.

(Llamaremos $R_{0}$ a cualquier subconjunto de $\mathbb{R}$ que tenga una cardinalidad igual a $\mathbb{N}$. Puede ser un subconjunto de $\mathbb{N}$, con únicamente elementos con finitos dígitos; o no serlo, cuando el conjunto puede contener únicamente hasta $\aleph_{0}$ elementos, y éstos poseer finitas o infinitas cifras cada uno: $\left|\mathrm{R}_{0}\right|=|\mathbb{N}|$ ).

Demostración: La definición de $\mathrm{R} \uparrow_{\aleph_{0}}$ implica que $\mathrm{R} \uparrow_{\aleph_{0}}$ contiene como elemento al antidiagonal cognado obtenido sobre el rango de $\mathrm{f}: \mathbb{N} \rightarrow \mathbb{R}$ ii, por ser este antidiagonal un elemento, un número real, que cumple, como se ha demostrado en el Teorema 1, con la restricción de poseer $\aleph_{0}$ dígitos. 
Por lo tanto, $\mathrm{R} \boldsymbol{T}_{\aleph_{0}}$ posee una cardinalidad mayor que $\mathbb{N}$, al incluir adicionalmente respecto a $\mathbb{N}$ a cualquier número implicado por el elemento antidiagonal cognado, que no puede ser $\aleph_{0}$-enumerado por $\mathbb{N}$ :

$$
\left|\mathrm{R}_{\boldsymbol{N}_{0}}\right|>|\mathbb{N}|
$$

Y como $R \uparrow_{\aleph_{0}}$ por definición es un subconjunto propio o impropio de $\mathbb{R} \mathbb{i}$, entonces $R \Upsilon_{\aleph_{0}}$ puede ser igual o menor al contradominio universo $\mathbb{R} i$ :

$$
\left|\mathbb{R}_{i i}\right| \geq\left|R_{N_{0}}\right|>|\mathbb{N}| \text {. }
$$

Ilustro el argumento, tratando de hacer lo más explícita posible la nueva idea aquí expuesta: el recorrido de la función diagonal sólo puede ocurrir, por su misma definición, sobre una cantidad $\aleph_{0}$ de números $r_{i} \in \mathbb{R} i i$; por ende, el número diagonal resultante sólo puede tener una cantidad $\aleph_{0}$ de cifras, al terminar, como se puede constatar en la Tabla 2 , en $a_{n n}, y$ no en $a_{n+1} n+1$, o en $a_{n+x} n+x$, ni en $a_{r i r i}$.

Insistimos: Es sólo cuando se mapean los números naturales $n \in \mathbb{N}$ del dominio (cada uno por definición con una cantidad finita de dígitos) hacia un conjunto de números reales $\mathbb{R} i{ }_{N} \subseteq \mathbb{R} i i$ en el rango (con $\left|\mathbb{R} i_{\mathbb{N}}\right|=\aleph_{0}$, debido a ser la cardinalidad hasta $r_{i}$ tomada desde el principio como no necesariamente mayor que la cardinalidad $\mathbb{N}$ ) (y por tener cada uno de los $r_{i} \in \mathbb{R} i i$ una serie de cifras que pueden ser finitas o infinitas), que uno obtiene la conclusión, con el proceso de la diagonal, de que hay al menos algún número real, el de la antidigonal, que no puede ser $\aleph_{0}$-enumerado, y por lo tanto que $\exists R_{1}$ tal que $\left|R_{1}\right|>\left|R_{0}\right|$.

Lema 2. (Una obviedad aquí, pero antecedente argumentativo importante para los menos obvios raciocinios en los teoremas demostrados más adelante, como ya se verá).

Cualquier número $\mathrm{r}_{1} \in \mathrm{R}_{1}$ tiene que ser un número real. No hemos ido más allá de los $\mathrm{r}_{\mathrm{i}}$ $\in \mathbb{R}$ ii o de los $r \in \mathbb{R}$. 
Demostración. Ello sucede o bien porque $\mathrm{r}_{1} \in \mathrm{R}_{0}$, o bien por corresponder necesariamente $\mathrm{r} 1$ a un diagonal cognado, y no a un diagonal ajeno. Hecho que le permitió concluir a Cantor que $|\mathbb{R} i|>|\mathbb{N}|$; pues de lo contrario, con un diagonal y un antidiagonal ajenos, por definición, se obtendría un número x tal que no estaría garantizado lógicamente que

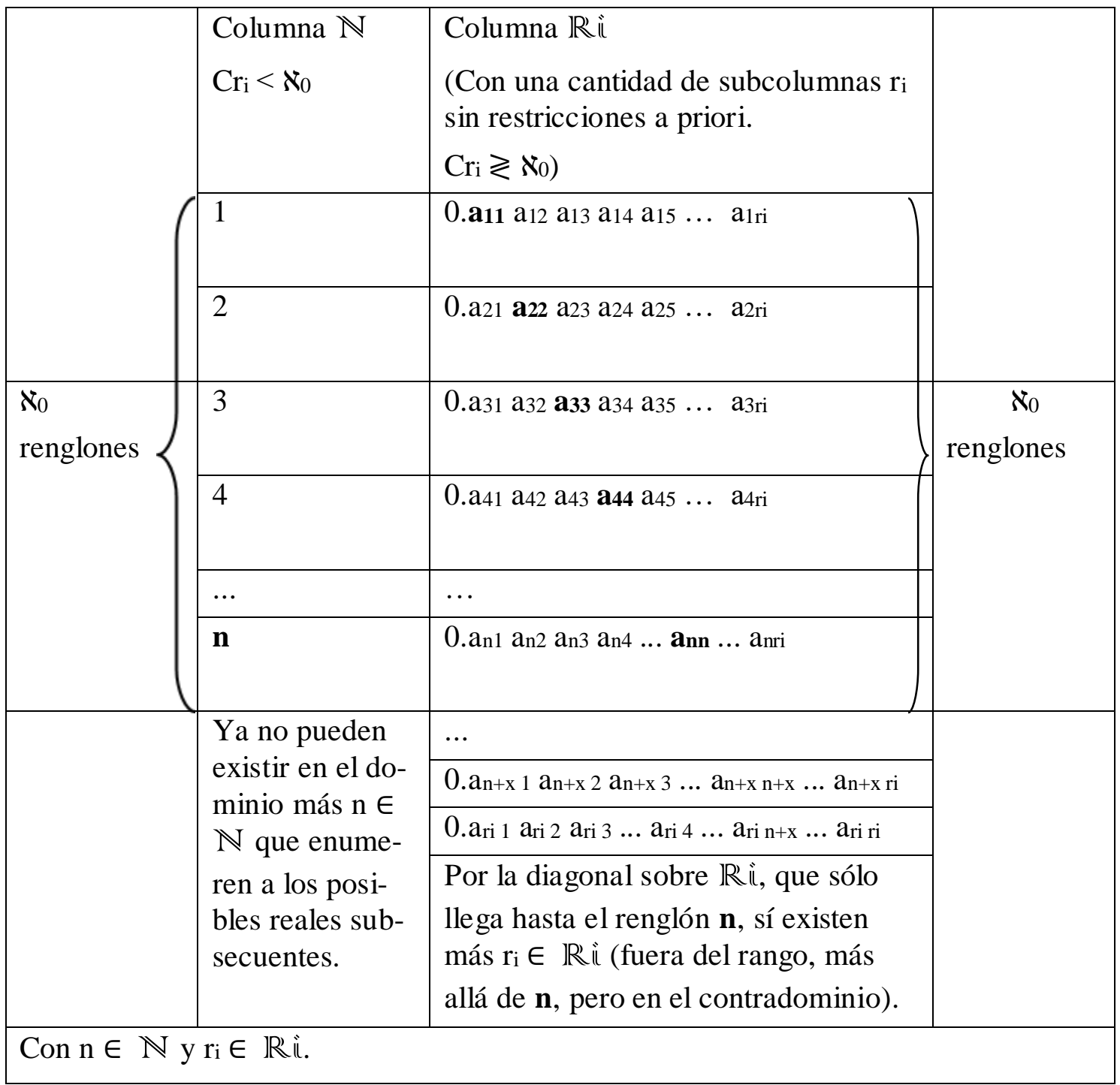

TABLA 2. f: $\mathbb{N} \rightarrow \mathbb{R}$ ii.

$\mathrm{x} \in \mathbb{R}$ i $\mathrm{y}$ por lo tanto en el razonamiento de Cantor no se estaría demostrando que $\mathrm{x}$ fuera algún número real del que se pudiera deducir la mayor cardinalidad de $\mathbb{R} i i$; y por ende su prueba estaría equivocada.

Consecuentemente:

Corolario 1. El primer $\aleph$ obtenible a partir del proceso de la diagonal de Cantor sobre $\mathbb{R}^{R}$ ii, es $\left|\mathrm{R}_{\aleph_{0}}\right| \equiv\left|\mathrm{R}_{1}\right|=\aleph_{1} \leq \mid \mathbb{R}$ ii $\mid$.

Se recalca, es llamado $\left|R_{1}\right|$ ó $\aleph_{1}$ por ser el primer número cardinal generado por el proceso de la diagonal de Cantor desde $\mathrm{f}: \mathbb{N} \rightarrow \mathbb{R}$ ii. Sin presuponer que a partir de otro procedimiento, o como axioma, pueda o no pueda obtenerse un $N$ intermedio. 
Corolario 2. Por lo tanto, hasta aquí, para que $\aleph_{1}$ fuera igual a la cardinalidad de $\mathbb{R}$ ¿i, i.e. a la cardinalidad de $\mathbb{R}$, sería necesario que la cantidad máxima de cifras que componen a cualquier número real fuera $\aleph_{0}$ :

$\aleph_{1}=|\mathbb{R} i| \leftrightarrow \forall \mathrm{r}_{i} \in \mathbb{R} i i\left|\mathrm{Cr}_{\mathrm{i}}\right| \leq \aleph_{0}$. O, lo que es lo mismo,

$\aleph_{1}=|\mathbb{R}| \leftrightarrow \forall r \in \mathbb{R}|\operatorname{Cr}| \leq \aleph_{0}$. Con $\aleph_{1}=\left|R_{1}\right|$.

Surge la pregunta: ¿| $|\mathbb{R} i|=\left|R_{\aleph_{0}}\right|$ ? ¿Acaso no existen números reales con más de $\aleph_{0}$ cifras, cuando ya sabemos que existe la cantidad $\left|R_{1}\right|=\aleph_{1}$ ?

Para determinarlo, se expone lo siguiente.

\subsection{AMPLIACIÓN DEL ARGUMENTO DE LA DIAGONAL DE CANTOR SOBRE RRi}

Antes de arribar a una prueba más general, que amplía el alcance del proceso de la diagonal, primero se procede a demostrar el siguiente teorema. Con el fin de destacar lo más claramente posible los argumentos, se han hecho explícitas las pruebas que son similares a otras demostraciones desarrolladas anteriormente.

Teorema 3: El conjunto $\mathbb{R}$ ¿i de los números reales en el intervalo $[0,1)$, cuyos elementos $r_{i}$ tienen cualquier número de cifras cada uno en su desarrollo decimal, sin restricciones previas al respecto, posee una cardinalidad no igual y estrictamente mayor que el subconjunto $R \uparrow_{N_{0}}$ de los números reales que tienen una restricción tal que sus elementos $r_{i}$ están limitados a estar constituidos cada uno por un número máximo de $\aleph_{0}$ cifras:

$$
\left|\mathbb{R}_{i i}\right|>\left|\operatorname{RiN}_{N_{0}}\right|(>|\mathbb{N}|) \text {. }
$$

Es decir $\left|\mathbb{R}^{R} i\right|>\left|R_{l}\right|\left(>\left|\mathrm{R}_{0}\right|\right)$.

Demostración. (Con el procedimiento de la diagonal). Investiguemos si $\left|\mathrm{R}_{\Upsilon_{\aleph_{0}}}\right|=|\mathbb{R} i \mathrm{i}|$. Es decir, si f: $\mathrm{R}_{\boldsymbol{N}_{0}} \rightarrow \mathbb{R}$ ii es biyectiva. Formamos a la manera de Cantor una correspondencia, que en este caso relaciona a $\mathrm{R} \uparrow_{\aleph_{0}}\left(\equiv \mathrm{R}_{1}\right)(\mathrm{y}$ ya no sólo a $\mathbb{N})$ en el dominio, con $\mathbb{R} \mathfrak{i}$ en el contradominio.

Apliquemos la diagonal en $\mathbb{R} i i$, a la cual en este caso llamaremos 1-ampliación de la diagonal de Cantor sobre $\mathbb{R} \mathbb{R}^{\prime} i$ :

En la función $\mathrm{f}: \mathrm{R} \boldsymbol{N}_{\boldsymbol{N}_{0}} \rightarrow \mathbb{R}$ i $i$ a cada cifra de la parte decimal de cada $\mathrm{r}_{i} \in \mathbb{R}$ ii, se le representa como apq, donde $\mathrm{p}$ indica el lugar que ocupa en la enumeración el $\mathrm{r}_{\mathrm{i}}$ al que pertenece la cifra respectiva; y q el lugar que ocupa el mismo dígito dentro de tal $\mathrm{r}_{\mathrm{i}}$ (de aquí se deriva que podamos realizar un arreglo como en la Tabla 3, donde p y q corresponden respectivamente al renglón y la columna en que tal dígito está ubicado).

Luego, sobre el conjunto $\mathbb{R} i i$ es operada una función diagonal $f_{d}=\{(p, q) \in A \times A: p=q\}$ que selecciona de cada $r_{i}$ a una cifra tal que $p=q$, con lo cual se forma un número real diagonal 0. a11 a22 a33 a $44 \ldots$ ann $\ldots$ arrr1 .

Desde ese real diagonal se genera un número real antidiagonal $r_{a} \in[0,1)$ (por ejemplo, estableciendo una función característica que sustituye sobre el real diagonal a cualquier dígito que difiera de 1 por $1 \mathrm{y}$ a toda cifra igual a 1 por 2).

Antidiagonal $r_{a}$ que difiere de cualquiera de los números en el rango $\mathbb{R} i \dot{i}$, es decir, que no pertenece a este rango inyectado desde el dominio $R \uparrow_{\aleph_{0}}$ de la función $\mathrm{f}: \mathrm{R} \uparrow_{\aleph_{0}} \rightarrow \mathbb{R}$ ii. 


\begin{tabular}{|c|c|c|}
\hline & $\mathrm{R} \uparrow_{\aleph_{0}} \equiv \mathrm{R}_{1}$ & Rii \\
\hline & $\begin{array}{l}\left|\mathrm{R}_{1}\right|=\aleph_{1} ; \text { con un límite } \\
\text { máximo transfinito } \aleph_{0} \text { de } \\
\text { cifras en cada } r_{i} \in \mathbb{R} \hat{i} \text {, es } \\
\text { decir, }\left|\mathrm{Cr}_{i}\right| \leq \aleph_{0}\end{array}$ & $\begin{array}{l}\text { Sin suponer a priori un límite transfinito de } \\
\text { cifras en cada } r_{i} \in \mathbb{R} i, \text { es decir, }\left|\mathrm{Cr}_{i}\right| \gtrless \\
\aleph_{1} \text {. }\end{array}$ \\
\hline & $\begin{array}{l}\text { Por lo tanto, la columna } \mathrm{R}_{1} \\
\text { tiene a lo más } \aleph_{0} \text { subcolum- } \\
\text { nas }\end{array}$ & $\begin{array}{l}\text { La columna Rii tiene al menos } \aleph_{1} \text { subco- } \\
\text { lumnas }\end{array}$ \\
\hline & $0 . \mathrm{a}_{11} \mathrm{a}_{12} \mathrm{a}_{13} \mathrm{a}_{14} \ldots \mathrm{a} \mathrm{a}_{10}$ & $0 . a_{11} a_{12} a_{13} a_{14} \ldots$ a $1 n \ldots$ a $1 r_{i}$ \\
\hline & $0 . a_{21} a_{22} a_{23} a_{24} \ldots a_{2 r}$ & $0 . a_{21} \mathbf{a}_{22} a_{23} a_{24} \ldots$ a $2 n \ldots$ a $r_{i}$ \\
\hline & $0 . a_{31}$ a32 a33 a34 ... a3ro & $0 . a_{31} a_{32} \mathbf{a} 33$ a $34 \ldots$ a $3 n \ldots$ a $3 r_{i}$ \\
\hline & $0 . a_{41} a_{42} a_{43} a_{44} \ldots$ a $4 r_{0}$ & $0 . a 41$ a42 a 43 a $44 \ldots$ a $4 n \ldots$ a $4 r_{i}$ \\
\hline & $\ldots$ & $\ldots$ \\
\hline & 0. an 1 an2 an 3 an $4 . .$. anro & 0. an 1 an2 an 3 an $4 \ldots$ ann $\ldots$ anri \\
\hline & $\ldots$ & $\ldots$ \\
\hline $\begin{array}{l}\text { Renglón } \\
\mathbf{r}_{1}\end{array}$ & $0 . a_{r 11} a_{r 12} a_{r_{1} 3} a_{r 14} \ldots$ ariro & $0 . \operatorname{ar}_{11} \operatorname{ar}_{12} \operatorname{ar}_{13} \operatorname{ar}_{14} \ldots a_{r_{1} r_{1} \ldots} \operatorname{ar}_{1 r_{i}}$ \\
\hline & Ya no pueden existir en el & \\
\hline & dominio más $r_{i} \in R_{1}$ que $\aleph_{1^{-}}$ & $0 . \operatorname{ar}_{i 1} \operatorname{ari}_{2} 2 \operatorname{ar}_{i} 3 \operatorname{ar}_{i} 4 \ldots$ ariri \\
\hline & $\begin{array}{l}\text { enumeren a los posibles } \\
\text { reales subsecuentes en } \mathbb{R} i i\end{array}$ & $\begin{array}{l}\text { Por el proceso diagonal, que sólo llega, por } \\
\text { hipótesis, hasta el renglón } \mathbf{r}_{1} \text {, sí existen } \\
\text { más } r_{i} \in \mathbb{R} \text { ii tales que } r_{i} \notin R_{1}\end{array}$ \\
\hline Donde $n \in$ & $\mathbb{N}, r_{0} \in \mathrm{R}_{0}, \mathrm{r}_{1} \in \mathrm{R}_{1}, \mathrm{r}_{\mathrm{i}} \in \mathbb{R} i \mathrm{i}, \mathrm{a}$ & $\mathrm{r}_{\mathrm{i}} \geq \operatorname{ar} \mathrm{ar}_{0} \mathrm{y}\left|\mathrm{Cr}_{\mathrm{i}}\right| \geq\left|\mathrm{Cr}_{1}\right|$ \\
\hline $\begin{array}{l}\text { Y cada ro } \\
\aleph_{0}(=\text { la ca } \\
\text { ahora a pa }\end{array}$ & $\begin{array}{l}\text { la segunda posición represer } \\
\text { tidad máxima posible de cifra } \\
\text { ir de la diagonal de Cantor). }\end{array}$ & $\begin{array}{l}\text { la cantidad creciente de columnas hasta } \\
\text { de esos números reales demostrada hasta }\end{array}$ \\
\hline $\begin{array}{l}\text { Asimismo, } \\
\text { nes hasta } \\
\text { subcolumn } \\
\text { Por lo tant } \\
\text { real puede } \\
\text { conjunto d } \\
\text { ser igual o }\end{array}$ & $\begin{array}{l}\text { cada } \mathrm{r}_{1} \text { en la primera posiciór } \\
\text { correspondientes a } \mathrm{R}_{1} \text {. Y ri } 1 \\
\text { s correspondientes a } \mathbb{R} i \mathrm{i} \text {. } \\
\text { en la columna } \mathrm{R}_{1} \text { del domini }\end{array}$ & $\begin{array}{l}\text { epresenta la cantidad creciente de renglo- } \\
\text { presenta la posible cantidad creciente de } \\
\text { la mayor cantidad de cifras que un número } \\
\text { arır. Mientras que en la columna Rii del } \\
\text { respectiva (arriri) queda de inicio abierta a } \\
\text { arıro. }\end{array}$ \\
\hline
\end{tabular}

TABLA 3. f: $\mathrm{R} \Gamma_{\mathrm{N}_{0}} \rightarrow \mathbb{R}$ ii.

Probando con eso que si bien hay inyección, no hay suprayección y por lo tanto, que $\mathbb{R}$ ii es un infinito mayor que $\mathrm{R} \uparrow_{N_{0}}$.

Demostrando que en este caso los $r_{i} \in \mathbb{R}$ i $i$ como conjunto de imágenes de $R \uparrow_{\aleph_{0}}$ en $\mathbb{R}$ i i son menos en cardinalidad que los $r_{i} \in \mathbb{R} i i$ como contradominio universal; que contradominio $>$ rango (y por lo tanto $r_{i}>r_{0}$ ).

Llamaremos $R_{2}$ a ese subconjunto infinito determinado del contradominio $\mathbb{R}$ ii, mayor que $R_{1}$. $R_{2}$ es o un subconjunto propio o igual a $\mathbb{R} i i$, esto aún no lo establecemos.

Por lo tanto se deduce: 
Corolario 3. $\exists R_{2} \subseteq \mathbb{R}$ ii tal que $\left|R_{2}\right|>\left|R_{1}\right|$. Con $\left|R_{2}\right|=\aleph_{2}$.

$\left|R_{2}\right|=\aleph_{2}$ indica aquí el hecho de que $\left|R_{2}\right|$ es el segundo $\mathbb{\aleph}$ obtenido a partir de $f: \mathbb{N}$ $\rightarrow \mathbb{R}$ i a través de la aplicación recursiva del método de la diagonal, sin presuponer que a partir de otro procedimiento pueda o no pueda obtenerse otro $\aleph$ intermedio.

\subsection{CONSTRUCCIÓN DE LA EXTENSIÓN DE DÍGITOS EN LOS NÚMEROS REALES $r_{i} \in \mathbb{R} i i$}

La demostración con la 1-ampliación de la diagonal de Cantor tiene la limitación de únicamente permitir concluir que se justifica lógicamente la existencia y construcción de números reales exclusivamente hasta $\aleph_{1}$ dígitos, mismos que integran al conjunto $\mathrm{R}_{2}$.

Puede haber una cantidad mayor que esa de dígitos en un número real, pero su existencia aún no la hemos verificado de manera matemática o lógica. Lo cual haremos más adelante.

Teorema 4. Además de ser mayor a $R_{1}$, el conjunto $R_{2}$ obtenido por el proceso 1-diagonal está integrado de forma tal que sus elementos $r_{i}$ son cada uno un número real, que consta a lo máximo de una cantidad de $\aleph_{1}$ cifras. $Y R_{2}$ tiene una cardinalidad menor o igual a la de $\mathbb{R}^{\mathrm{R}}$ il:

$$
\begin{aligned}
& \mathrm{R}_{2} \equiv \mathrm{R} \uparrow_{\aleph_{1}}=\left\{\mathrm{r}_{\mathrm{i}} \in \mathbb{R} i \mathrm{i}|| \mathrm{Cr}_{\mathrm{i}} \mid \leq \aleph_{1}\right\} \subseteq \mathbb{R} i ; y \\
& \left|\mathbb{R}^{2} i\right| \geq\left|\mathrm{R}_{2}\right|
\end{aligned}
$$

Demostración: En la función $\mathrm{f}: \mathrm{R}_{1} \rightarrow \mathbb{R}$ ii la diagonal cognada sobre $\mathbb{R}$ ii sólo puede tener una cantidad de cifras igual a la de $\left|\mathrm{R}_{1}\right|$ porque, por hipótesis, es trazada sobre una cantidad de números reales en $\mathbb{R} i i$ igual a la cantidad de los números reales que los $\aleph_{1}$-enumeran desde la columna del dominio $\mathrm{R}_{1}$, pues, por su construcción, la diagonal sobre el conjunto de imágenes de $R_{1}$ en $\mathbb{R} i i$ (conjunto al que llamaremos $\mathbb{R}^{\prime i} \mathrm{R}_{1}$ ) selecciona un dígito de cada número real que es elemento de este conjunto. Esto es, el recorrido de la diagonal sólo puede ocurrir sobre una cantidad $\aleph_{1}$ de cifras.

No puede ser operada sobre un conjunto con más números reales que $\aleph_{1}$, pues eso sería una petición de principio. Y es por no ser una petición de principio que son válidos e importantes los resultados previos (incluido el original de Cantor de $\mid \mathbb{R}$ i $i|>| \mathbb{N} \mid$; y también el de que existe al menos un número real, correspondiente a la antidiagonal, que no puede ser $\aleph_{1}$-enumerado, y por lo tanto que $\exists R_{2}>R_{1}>\mathbb{N}$ ).

A partir del conjunto formado por el rango $\mathbb{R}^{i} \mathrm{i}_{1}$ correspondiente al dominio $\mathrm{R}_{1}$, es construido un subconjunto $\mathrm{R} \boldsymbol{N}_{\mathrm{N}_{1}} \subseteq \mathbb{R} i \mathrm{i}$ (que puede ser igual o propio), con la restricción de que sus elementos sólo pueden poseer entre una y $\aleph_{1}$ cifras (por el Axioma Esquema de Separación).

El conjunto $\mathrm{R}_{\aleph_{1}}$ no solamente contiene a los elementos del conjunto $\mathbb{R} i \mathrm{i}_{1}$. A este último le podemos agregar (por el Axioma de Unión) los elementos del conjunto $\mathrm{R}_{\mathrm{a}}$ formado (aplicando el Axioma Esquema de Separación) por cualquier número real implicado por la antidiagonal sobre $\mathbb{R}$ ii. Mismos elementos que cumplen también, como lo acabamos de demostrar, con la restricción estipulada $\left|\mathrm{Cr}_{i}\right| \leq \aleph_{1}$.

Es así que se determina cómo está conformado el nuevo conjunto $R_{2}=\mathbb{R}^{2} \mathbb{R}_{1} \cup R_{a}$, el cual igualmente es llamado $\operatorname{R}_{\aleph_{1}}=\left\{r_{i} \in \mathbb{R} i i|| \mathrm{Cr}_{i} \mid \leq \aleph_{1}\right\} \subseteq \mathbb{R} i i$. 
Y como $R_{2}$ por definición es un subconjunto, propio o igual, de $\mathbb{R}$ ii, entonces $\mathbb{R} i i$ no puede ser menor que $R_{2}$ y consiguientemente, además de ser $R_{2}$ mayor a $R_{1}$, no necesariamente $R_{2}=\mathbb{R}$ ii, pues sigue viva la opción de que $R_{2}$ sea menor al contradominio universal Rii:

$$
|\mathbb{R} i i| \geq\left|R_{2}\right|>\left|R_{1}\right| \text {. }
$$

Se puede apreciar, entonces, que Cantor y la matemática conjuntista después de él, cometieron el equívoco de identificar al que hemos llamado $\mathrm{R}_{1}$, el único conjunto justificado lógicamente por el proceso de la diagonal en el razonamiento original de Cantor, con $\mathbb{R} i i$ y con $\mathbb{R}$. Ni siquiera con $R_{2}$ se ha ido más allá de $\mathbb{R}$ ï ni de $\mathbb{R}$ :

Teorema 5: Cualquier número $r_{2} \in R_{2}$ tiene que ser un número real $r_{i} \in \mathbb{R}^{\prime} i$ :

$\mathrm{r}_{2} \in \mathrm{R}_{2} \rightarrow \mathrm{r}_{2} \in \mathbb{R}$ ii.

Demostración: Ello ocurre:

1) $O$ bien porque $r_{2}$ sea un $r_{i} \in R_{1}$,

2) O bien, por ser obtenido r2 necesariamente como un diagonal cognado (definición 1), y no como un diagonal ajeno. En donde se toma (por definición del procedimiento diagonal) una cifra $r_{i} \in \mathbb{R} i i$ - por ejemplo, de 0 a 9 en el sistema decimal - de cada número real en el rango; siendo por ende el número diagonal cognado obtenido un número real. Que genera un número antidiagonal cognado, el cual por definición consta únicamente de dígitos, por ejemplo, de las cifras 1 y 2, que lo conforman como otro número real.

Esto es, $\mathrm{r}_{2}$ es un número real por no ser obtenido como un diagonal ajeno, hecho lógicamente equivalente al que le permitió concluir anteriormente a Cantor que $|\mathbb{R} i|>|\mathbb{N}|$. Pues de lo contrario, con un diagonal ajeno, por definición, se obtendría un número x tal que no se estaría garantizando lógicamente que $\mathrm{x} \in \mathbb{R}$ i $\mathrm{y}$ por lo tanto no se estaría demostrando que $\mathrm{x}$ es algún número real que contribuye a incrementar la cardinalidad de Rii.

\subsection{LA RENORMALIZACIÓN DEL CONJUNTO POTENCIA P( $\mathbb{N})$.}

A partir del descubrimiento de la diferencia en cuanto a cardinalidad entre $R_{1}$ y $\mathbb{R} i \dot{i} \geq R_{2}$, procederé a continuación a redimensionar los alcances de distintos conjuntos infinitos, como lo son $\mathrm{R}_{1}, \mathrm{P}(\mathbb{N})$ y $\mathbb{R}$ ii, lo cual se facilita por el hecho de corregir y adaptar para $\mathrm{R}_{1}$ una demostración ya existente en la Teoría de Conjuntos respecto a $\mathbb{R}$ ii, pero que confunde a $\mathrm{R}_{1}$ con $\mathbb{R}$ ii.

Lema 3. Sea $\mathrm{C}_{\mathrm{Ns}}$ la cantidad de elementos (números naturales) que pertenecen a cada conjunto $N_{s} \subseteq \mathbb{N}$. Entonces $\left|\mathrm{C}_{\mathrm{Ns}}\right| \leq \aleph_{0}$.

Demostración. $\mathbb{N}$ tiene a lo más $\aleph_{0}$ elementos, por definición de la cardinalidad de $\mathbb{N}$. Por lo tanto, cada subconjunto $\mathrm{N}_{\mathrm{s}}$ de $\mathbb{N}$ tiene a lo más $\aleph_{0}$ elementos: $\left|\mathrm{C}_{\mathrm{Ns}}\right| \leq \aleph_{0}$. Por el Axioma Esquema de Separación.

Usaremos el Teorema de Schroeder-Bernstein para probar, con la siguiente igualdad, que la cardinalidad de $P(\mathbb{N})$ es equivalente a la de $R 1$, y no a la de $\mathbb{R}$ ii:

Teorema 6. $\left|R_{1}\right|=|P(\mathbb{N})|$.

Demostración.

1. Probamos primero $\left|R_{1}\right| \leq|P(\mathbb{N})|$. 
Se establece una función $\mathrm{f}: \mathrm{R}_{1} \rightarrow \mathrm{P}(\mathbb{N})$, donde es inyectado cada $\mathrm{r}_{1} \in \mathrm{R}_{1} \subset(0,1)$ hacia cada $\mathrm{N}_{\mathrm{s}} \subseteq \mathbb{N}$, con $\mathrm{N}_{\mathrm{s}} \in \mathrm{P}(\mathbb{N})$.

Se escribe la parte decimal de cada $r_{1} \in R_{1}$ en forma binaria, como una secuencia de dígitos 0 y 1 que, por el Lema 1, sólo puede tener una cardinalidad de cifras menor o igual a $\aleph_{0}:\left|\mathrm{Cr}_{\mathrm{i}}\right| \leq \aleph_{0}$.

Estipulamos una correspondencia tal que el n-ésimo dígito 1 dentro de cada real $\mathrm{r}_{\mathrm{i}}$ indica que el número natural $\mathrm{n}$ sí pertenece a $\mathrm{N}_{\mathrm{s}}$, mientras que el $\mathrm{n}$-ésimo dígito 0 indica que $\mathrm{n}$ $\notin \mathrm{N}$ s.

Previamente ya se sabe que, para evitar repeticiones, en caso de generar el mismo número binario más de una vez, tomamos sólo aquella forma decimal que no termine únicamente en ceros. Y que, aunque $(0,1)$ no incluya a 0 , que se corresponde con el conjunto vacío, $|(0,1)|=|[0,1)|$.

Por ello, cada $\mathrm{r}_{1} \in \mathrm{R}_{1}$ corresponde a un diferente subconjunto $\mathrm{N}_{\mathrm{s}}$ de $\mathbb{N}$, al ocurrir que $\left|\mathrm{C}_{\mathrm{Ns}}\right| \leq \aleph_{0}$ (por el Lema 3) y $\left|\mathrm{Cr}_{\mathrm{i}}\right| \leq \aleph_{0}$, lo cual implica que cualquier $\left|\mathrm{C}_{\mathrm{Ns}}\right|$ puede ser igualada por alguna $\left|\mathrm{Cr}_{\mathrm{i}}\right|$ :

$\forall \mathrm{N}_{\mathrm{s}} \exists \mathrm{r}_{\mathrm{i}}$ tal que $\left|\mathrm{C}_{\mathrm{Ns}}\right|=\left|\mathrm{Cr}_{\mathrm{i}}\right|$.

Y por lo tanto, no hay ningún $\mathrm{N}_{\mathrm{s}}$ que no pueda ser abarcado en su cantidad de elementos por un $r_{1}$ con semejante cantidad de cifras. De modo que $\left|R_{1}\right| \leq|P(\mathbb{N})|$.

2. Se demuestra $|P(\mathbb{N})| \leq\left|R_{1}\right|$.

Cada subconjunto de $\mathbb{N}$ corresponde, como se estableció en la parte 1 de esta prueba, a un infinito encadenamiento binario de las cifras 0 y 1 , de manera que se puede inyectar tal cadena al $\mathrm{r} 1$ número decimal correspondiente. De esta manera, cada subconjunto $\mathrm{N}_{\mathrm{s}} \in$ $\mathrm{P}(\mathbb{N})$ corresponde a un diferente decimal $\mathrm{r}_{1} \in \mathrm{R}_{1}$ conformado exclusivamente por los dígitos 0 y 1 , con $\left|\mathrm{Cr}_{1}\right| \leq \aleph_{0}$. De aquí, tenemos $|\mathrm{P}(\mathbb{N})| \leq\left|\mathrm{R}_{1}\right|$.

Por lo tanto, por 1 y 2 en esta prueba y el Teorema de Schroeder-Bernstein, $|P(\mathbb{N})|=$ $\left|\mathrm{R}_{1}\right|$, es decir, existen exactamente tantos números reales en $\mathrm{R}_{1}$ como hay subconjuntos de los naturales en $\mathrm{P}(\mathbb{N})$.

Teorema 7. $\left|R_{1}\right|=2^{\mathrm{N}_{o}}$

Demostración.

$\left|\mathrm{R}_{1}\right|=|\mathrm{P}(\mathbb{N})|$ (por el Teorema 6$)$.

$|\mathrm{P}(\mathbb{N})|=2^{\aleph_{0}}$ (por un corolario subsecuente al Teorema de Cantor).

$\left|\mathrm{R}_{1}\right|=2^{\mathrm{N}_{0}}$ (por transitividad).

Teorema 8. $\left|\mathbb{R}^{R} \ddot{i}\right|>|P(\mathbb{N})|$.

Demostración.

$|\mathbb{R} i|>\left|R_{1}\right|$ (por el teorema 3 ).

$\left|R_{1}\right|=|P(\mathbb{N})|$ (por el teorema 6).

$|\mathbb{R} i|>|P(\mathbb{N})|$ (por transitividad). 
Dicho de otra forma: La cantidad de elementos $\mathrm{N}_{\mathrm{s}} \in \mathrm{P}(\mathbb{N})$ está limitada por la cardinalidad $\aleph_{0}$ (por el Lema 3), mientras que $\mathbb{R} i i$ contiene, además de los reales ri que forman un conjunto con cardinalidad $\mathrm{R}_{1}$ por el Lema 1 (que se corresponden biyectivamente con esos elementos $\mathrm{N}_{\mathrm{s}}$ por el Teorema 6 ), a aquellos otros $\mathrm{r}_{\mathrm{i}}$ que poseen más de $\boldsymbol{\aleph}_{0}$ cifras (por el teorema 4) y que, por lo tanto, forman al menos un conjunto con cardinalidad mayor.

Corolario 4: Para que ocurriese $|\mathbb{R} i i|=\left|R_{2}\right|=\aleph_{2}$, sería necesario que la cantidad máxima posible de cifras de un número real fuera $\aleph_{1}$ :

$$
|\mathbb{R} i|=\left|R_{2}\right| \leftrightarrow\left|C_{i}\right| \leq \aleph_{1} \text {. Con }\left|R_{2}\right|=\aleph_{2} \text {. }
$$

Surge la pregunta: ¿ $\mathbb{R} \hat{i} i=\mathrm{R}_{\aleph_{1}}$ ? ¿Acaso no existen números reales con más de $\aleph_{1}$ cifras, cuando ya sabemos que existe el cardinal $\left|R_{2}\right|=\aleph_{2}$ ?

O, más en general: ¿ $\mathbb{R} i i=R \aleph_{\aleph_{\alpha}}$ ? ¿Acaso no existen los cardinales $\left|\mathrm{R}_{\alpha}\right|=\aleph_{\alpha} \mathrm{y}\left|\mathrm{R}_{\alpha+1}\right|=$ $\aleph_{\alpha+1}$. Y por lo tanto, acaso no existen siempre números reales con más de $\aleph_{\alpha}$ cifras?

4. GENERALIZACión DEL ARGUMENTO DE LA DiAgONAL DE CANTOR SOBRE LOS R $\boldsymbol{\alpha}$ SUBCONJUNTOS TRANSFINITOS DE RRi.

A continuación demostraremos inductivamente el caso más general, en el cual $\alpha$ implica $\alpha+1$.

Para ello argumentamos que, si suponemos la existencia de un conjunto

$\mathrm{R}_{\alpha} \equiv \mathrm{R} \uparrow_{\boldsymbol{N}_{\alpha-1}}=\left\{\mathrm{r}_{\mathrm{i}} \in \mathbb{R} \boldsymbol{R} i|| \mathrm{Cr}_{\mathrm{i}} \mid \leq \boldsymbol{\aleph}_{\alpha-1}\right\}$,

entonces necesariamente, por el procedimiento de la diagonal, se deduce que existe un conjunto

$\mathrm{R}_{\alpha+1} \equiv \mathrm{R} \uparrow_{\aleph_{\alpha}}=\left\{\mathrm{r}_{\mathrm{i}} \in \mathbb{R} \mathrm{R} i|| \mathrm{Cr}_{\mathrm{i}} \mid \leq \aleph_{\alpha}\right\}$

tal que
$\mathrm{R}_{\alpha} \subset \mathrm{R}_{\alpha+1} \wedge\left|\mathrm{R}_{\alpha}\right| \supseteqq\left|\mathrm{R}_{\alpha+1}\right|<\left|\mathbb{R}_{i} i\right|$.

Esto es, dado un conjunto $\mathrm{R}_{\alpha} \subseteq \mathbb{R}^{i} i$ con $\aleph_{\alpha}$ cantidad de números reales como elementos, su ordenación cantoriana como dominio, por ejemplo en una columna, sólo puede alcanzar la cantidad $\aleph_{\alpha}$ de ocurrencias o renglones.

Si se establece una correspondencia funcional $\mathrm{f}: \mathrm{R}_{\alpha} \rightarrow \mathbb{R}$ i $\mathrm{i}$ y se traza una diagonal que selecciona una cifra y sólo una de cada renglón del conjunto de las imágenes ri en $\mathbb{R}$ ii, esa diagonal sólo podrá tener por tanto, con base en el supuesto de partida, una cantidad de cifras igual a $\aleph_{\alpha}$.

Pero siendo el caso de un número diagonal cognado, ello implicará, aplicando el algoritmo de tipo cantoriano, necesariamente la formación de un número antidiagonal cognado con igual cantidad $\aleph_{\alpha}$ de cifras.

Lo que demuestra que existe al menos un número real antidiagonal $r_{a}$ tal que $r_{a} \notin R \alpha$. Ello permite deducir la existencia siempre de un conjunto $R_{\alpha+1} \subset \mathbb{R} i i$ como un contradominio mayor al conjunto del rango correspondiente a $R_{\alpha}$ en $\mathbb{R} i i$; con $|\mathbb{R} i i|>\left|R_{\alpha+1}\right|>\left|R_{\alpha}\right|$.

Más en detalle, las demostraciones con la diagonal de Cantor, y con sus ampliaciones argumentadas en este escrito, tienen la limitación lógica de únicamente permitir concluir que:

Teorema 9: Si suponemos la existencia de cualquier conjunto de números reales $R_{\alpha} \equiv$ $R \uparrow_{\aleph_{\alpha-1}}=\left\{r_{i} \in \mathbb{R}^{\prime i}|| C r_{i} \mid \leq \aleph_{\alpha-1}\right\}$, subconjunto de $\mathbb{R}\langle i i$, entonces necesariamente existe un conjunto $R_{\alpha+1}$ sucesor suyo cuya cardinalidad es mayor que la de $R_{\alpha} ; y$ los elementos de 
$R_{\alpha+1}$ son cada uno un número real con una cantidad a lo máximo de $\aleph_{\alpha}$ cifras en su composición decimal:

$$
\begin{aligned}
& \forall \alpha \exists R_{\alpha} \equiv R \uparrow_{\aleph_{\alpha-1}}=\left\{r_{i} \in \mathbb{R}^{\prime} \ddot{i}|| C r_{i} \mid \leq \aleph_{\alpha-1}\right\} \subseteq \mathbb{R}^{\prime} \ddot{i} \\
& \rightarrow \forall \alpha \exists R_{\alpha+1} \equiv R \uparrow_{\aleph_{\alpha}} \text { tal que }\left|R_{\alpha+1}\right|\left(=\aleph_{\alpha+1}\right)>\left|R_{\alpha}\right|\left(=\aleph_{\alpha}\right) \\
& \left.\wedge R_{\alpha+1}=\left\{r_{i} \in \mathbb{R}^{\prime} \ddot{i}|| C r_{i} \mid \leq \aleph_{\alpha}\right\}\right) .
\end{aligned}
$$

Demostración. (Con el procedimiento de la diagonal). Sea $\mathrm{f}: \mathrm{R}_{\alpha}\left(\equiv \mathrm{R} \boldsymbol{\Gamma}_{\boldsymbol{\alpha}-1}\right) \rightarrow \mathbb{R}$ ii. El mapeo de cada elemento de $\mathrm{R}_{\alpha}$ sobre $\mathbb{R} i i$ genera un conjunto de imágenes en $\mathbb{R}$ ii.

A cada cifra de la parte decimal de cada $r_{i} \in \mathbb{R} i i$ se le representa como apq, donde $p$ indica el lugar que ocupa en la $\aleph_{\alpha}$-enumeración el $r_{i}$ al que pertenece la cifra respectiva; y q el lugar que ocupa el mismo dígito dentro de tal $\mathrm{r}_{\mathrm{i}}$ (de aquí se deriva que podamos arreglar una Tabla como la número 4, donde p y q corresponden, respectivamente, al renglón y la columna en que tal dígito está ubicado).

Tracemos la función diagonal $\mathrm{f}_{\mathrm{d}}=\{(\mathrm{p}, \mathrm{q}) \in \mathrm{A} \times \mathrm{A}: \mathrm{p}=\mathrm{q}\}$ de Cantor sobre este rango en $\mathbb{R}$ ii (la $\delta$-ampliación de la diagonal de Cantor sobre $\mathbb{R} i i)$, que selecciona de cada $r_{i}$ a una cifra tal que $\mathrm{p}=\mathrm{q}$, con lo cual se forma un número real diagonal $0 . \mathrm{a}_{11} \mathrm{a}_{22} \mathrm{a}_{33} \mathrm{a}_{44} \ldots \mathrm{ar}_{\alpha} \mathrm{r}_{\alpha}$ (siendo a $r_{\alpha} r_{i}$ la cifra correspondiente al renglón $r_{\alpha}$ y a la subcolumna $r_{i} \geq r_{\alpha-1}$ ).

Desde ese real diagonal se genera un número real antidiagonal $r_{a} \in[0,1)$ (por ejemplo, estableciendo una función característica que sustituye sobre el diagonal a cualquier dígito que difiera de 1 por 1 y a toda cifra igual a 1 por 2 ).

Antidiagonal $r_{a}$ que difiere de cualquiera de los números en el rango $\mathbb{R} i i$, es decir, no pertenece a este rango inyectado desde el dominio $R_{\alpha}$ de la función $f: R_{\alpha} \rightarrow \mathbb{R} i$ i. Se demuestra con eso que si bien hay inyección, no hay suprayección y por lo tanto, que $\mathbb{R}$ ii es un infinito mayor que $\mathrm{R}_{\alpha}$.

Probando así que existe un conjunto $R_{\alpha+1}$ tal que $\left|R_{\alpha+1}\right|>\left|R_{\alpha}\right|$.

El diagonal cognado sobre el conjunto de imágenes de $\mathrm{R}_{\alpha}$ en el contradominio $\mathbb{R}^{2} i$ (al cual denotamos $\mathbb{R}_{i}\left(\mathbb{R}_{\alpha}\right)$ sólo puede tener una cantidad de cifras igual a la de $\left|R_{\alpha}\right|$. Entonces: codominio universo $\mathbb{R}_{i} i>$ rango $\mathbb{R}^{i}{ }^{i} \alpha$ (y por lo tanto, $\left.\mathrm{r}_{\mathrm{i}}>\mathrm{r}_{\alpha-1}\right)$.

Pues cualquier elemento que concierna al número diagonal cognado (y por ende cualquier elemento correspondiente al número antidiagonal cognado derivado de aquél, con un mapeo uno a uno de sus cifras) sólo puede tener en su composición una cantidad de dígitos igual a la cardinalidad de $\aleph_{\alpha}$. Ya que para su construcción por la diagonal, es seleccionada una cifra de cada número real que pertenece a ese conjunto de imágenes en $\mathbb{R} i i$.

Y ese número diagonal cognado es obtenido, por hipótesis de inicio, sobre un rango $\mathbb{R}^{\prime i} i_{\alpha}$ en tal columna $\mathbb{R} i i$ al que pertenece una cantidad de números reales igual a la cantidad de los números reales que los $\aleph_{\alpha}$-enumeran uno a uno desde la otra columna (el dominio $\mathrm{R}_{\alpha}$ ).

Aplicando el Axioma Esquema de Separación y el Axioma Unión, el rango $\mathrm{R}_{\alpha}$ forma por lo tanto, con cualquier número $r_{i}$ implicado por el diagonal cognado, un nuevo conjunto, al que llamaré $\mathrm{R} \uparrow_{\aleph_{\alpha}}\left(\equiv \mathrm{R}_{\alpha+1}\right)$, que tiene la restricción de que sus elementos únicamente pueden alcanzar una cantidad máxima $\aleph_{\alpha}$ de cifras:

$\mathrm{R}_{\aleph_{\alpha}} \equiv \mathrm{R}_{\alpha+1}=\left\{\mathrm{r}_{\mathrm{i}} \in \mathbb{R} \dot{i}|| \mathrm{Cr}_{\mathrm{i}} \mid \leq \aleph_{\alpha}\right\}$. 
Es claro que tal definición incluye a cualquier número $r_{i}$ implicado por el antidiagonal cognado, por ser éste un elemento que cumple, como se acaba de demostrar, con la restricción en cantidad de dígitos que determina al conjunto $\mathrm{R} \uparrow_{\aleph_{a+1}}$. Siendo por eso, recalcamos, que $R_{\alpha+1}$ posee una cardinalidad mayor que $R_{\alpha}$.

Y R $\uparrow_{\aleph_{a+1}}$, con su restricción, es un subconjunto (propio o igual) del contradominio Reii. Contradominio que no tiene restricciones en cuanto a la cantidad de cifras de sus elementos. Y puede tener una cardinalidad igual o menor a tal $\mathbb{R}$ ii.

Obviamente, como ya hemos demostrado la existencia de $\mathrm{R}_{1}$, con $\alpha=1$, y su cardinalidad igual a $\aleph_{1}$ a partir del proceso de la diagonal, entonces por los párrafos anteriores se deduce que $\exists R_{\alpha+1}=R_{1+1}=R_{2}$ tal que $\aleph_{2}=\left|R_{2}\right|>\left|R_{1}\right|=\aleph_{1}$. Y como así ahora obtenemos $\alpha$ $=2$, se sigue inmediatamente la existencia de $\left|R_{3}\right|>\left|R_{2}\right|$, y así sucesivamente.

Teorema 10. La clase $\mathbb{R}$ ii de los números reales en el intervalo $[0,1)$, cuyos elementos $r_{i}$ tienen cualquier número de cifras cada uno, sin restricciones previas al respecto, tiene como subconjunto propio a cualquier $\mathrm{R}_{\alpha}$ :

$\forall \alpha \mathrm{R}_{\alpha} \subset \mathbb{R} i \mathrm{i}$

Demostración. Sea $\mathrm{R}_{\alpha+1} \supseteq \mathrm{R}_{\alpha}$. Por definición incluye todos los elementos de los $\mathrm{R}_{\alpha}$ anteriores, pero además incluye a la antidiagonal cognada sobre $\mathrm{R}_{\alpha}$, la cual no pertenece a $\mathrm{R}_{\alpha}$, y por lo tanto, $\mathrm{R}_{\alpha} \subset \mathrm{R}_{\alpha+1}$.

Por otra parte, $\mathrm{R}_{\alpha+1} \subseteq \mathbb{R} i \mathrm{i}$, por provenir de $\mathrm{R} \alpha \mathrm{y}$ de su antidiagonal cognada.

Consecuentemente, $\mathrm{R}_{\alpha} \subset \mathrm{R}_{\alpha+1} \subseteq \mathbb{R} i$.

Por lo tanto, por transitividad, $R_{\alpha} \subset \mathbb{R} i i$.

Teorema 11. Rii posee una cardinalidad estrictamente mayor que cualquier subconjunto propio $R_{\alpha}$ de los números reales que tenga una restricción tal que sus elementos están limitados a estar constituidos, cada uno, por un número máximo de $\aleph_{\alpha-1}$ cifras:

$\forall \alpha\left|\mathbb{R}_{i}\right|>\left|R_{\alpha}\right|\left(\equiv\left|\operatorname{R}_{\kappa_{\alpha-1}}\right|\right)$.

Demostración. En la función $\mathrm{R}_{\alpha} \rightarrow \mathbb{R} i i, \mathrm{R}_{\alpha}$ da lugar, con su inyección, siempre a un rango $\mathbb{R}_{\mathbb{R} i \mathbb{R}_{\alpha}}$ cuya cardinalidad es menor a la del contradominio $\mathbb{R} i i$ del que es subconjunto, al ser siempre menor a un conjunto sucesor suyo $\mathrm{R}_{\alpha+1} \subseteq \mathbb{R} i i$, por el Teorema 9.

Esto es, el procedimiento diagonal nunca puede ser operado sobre todo el universo de la clase $\mathbb{R}$ ii en el contradominio de todos los números reales en el intervalo $[0,1)$, pues ello sería una petición de principio, ya que en la prueba siempre se parte como premisa de que cada elemento de $R_{\alpha}$ inyecta a un elemento de $\mathbb{R} i i$. Es decir, no hay justificación lógica para presuponer que $\left|\mathrm{R}_{\alpha}\right| \neq|\mathbb{R} i|$.

Y consistiría tal petición de principio en asumir que existe, antes de trazar la diagonal y su antidiagonal, al menos un número real $0 . a_{\mathrm{r}_{\mathrm{i}} 1} \mathrm{a}_{\mathrm{ri} 2} \mathrm{a}_{\mathrm{ri} 3} \mathrm{a}_{\mathrm{ri}} 4 \ldots \mathrm{a}_{\text {riri }}$ que rebasa al conjunto de imágenes de $\mathrm{R}_{\alpha}$ sobre $\mathbb{R} i i$, es decir presuponer que el contradominio es mayor que el rango. Que es tanto como presuponer que no existe suprayeccion antes de demostrar que la suprayeccion no existe.

Sólo sin la existencia de ese trazo universal de la función diagonal sobre toda la clase $\mathbb{R} i i$, es posible obtener los resultados previos (incluido el original de Cantor con $\alpha=0$ ) de que existe al menos un número real, el de la antidiagonal, que no puede ser $\aleph_{\alpha}$-enumerado.

Por lo tanto $\forall \mathrm{R}_{\alpha} \exists \mathrm{R}_{\alpha+1}$ tal que $\mid \mathbb{R}$ i $|>| \mathrm{R}_{\alpha+1}|>| \mathrm{R}_{\alpha} \mid$. Con $\left|\mathrm{R}_{\alpha}\right|=\aleph_{\alpha}$ y $\left|\mathrm{R}_{\alpha+1}\right|=$ $\aleph_{\alpha+1}$. 
Es decir, no existe un último subconjunto $\mathrm{R}_{\alpha}$ con cardinalidad igual a $\mathbb{R} i \mathrm{i}$. Parafraseando a Euclides, al referirse a los números primos: No existe un último número transfinito $|\mathrm{R}|(=\aleph)$, pues a cada número $|\mathrm{R}|$ le sigue otro número $|\mathrm{R}|$ mayor. No existe un último conjunto $\mathrm{R}_{\alpha}=\mathbb{R} \mathbf{i}$ con una cardinalidad última:

$\forall \alpha \neg \exists\left|\mathbf{R}_{\alpha}\right| \geq \mathbb{R} i$.

$\mathbb{R}$ ii es un contradominio infinito cuyo universo es mayor que cualquier subconjunto de rango $\mathrm{R}_{\alpha} \mathrm{y}$, consiguientemente (ver la Tabla 4 ), $\forall \mathrm{r}_{\alpha}, \mathrm{r}_{\mathrm{i}}, \mathrm{r}_{\alpha-1}<\mathrm{r}_{\mathrm{i}}$.

\begin{tabular}{|c|c|c|}
\hline & $\mathrm{R} \uparrow_{\aleph_{\alpha-1}} \equiv \mathrm{R}_{\alpha}$ & Rii \\
\hline & $\begin{array}{l}\text { Con una } \mathrm{Cr}_{i} \text { máxima de } \aleph_{\alpha-1} \\
\left|\mathrm{Cr}_{i}\right| \leq \aleph_{\alpha-1} \\
\left.\left(\mathrm{Y} \mid \mathrm{R}_{\alpha}\right]=\aleph_{\alpha}\right)\end{array}$ & $\begin{array}{l}\text { Sin suponer a priori un límite de ci- } \\
\text { fras en cada } r_{i} \in \mathbb{R} i{ }_{i} .\left|C r_{i}\right| \gtrless \aleph_{\alpha-1} \\
Y r_{i} \geq r_{\alpha-1} \text {. }\end{array}$ \\
\hline & $0 . a_{11} a_{12} a_{13} a_{14} \ldots$ a $1 r_{\alpha-1}$ & $0 . \mathbf{a} 11$ a 12 a13 a $14 \ldots$ a 1 ri \\
\hline & $0 . a_{21} a_{22} a_{23} a_{24} \ldots$ a $2 r_{\alpha-1}$ & $0 . a_{21}$ a22 a 23 a $24 \ldots$ a $2 r_{i}$ \\
\hline \multirow{2}{*}{$\begin{array}{l}\aleph_{\alpha} \text { renglo- } \\
\text { nes }\end{array}$} & $0 . a_{31} a_{32} a_{33} a_{34} \ldots$ a $3 r_{\alpha-1}$ & $0 . a_{31} a_{32} \mathbf{a} 33$ a34 $\ldots$ a $3 r_{i}$ \\
\hline & $0 . \mathrm{a} 41 \mathrm{a} 42 \mathrm{a} 43 \mathrm{a} 44 \ldots \mathrm{a} 4 \mathrm{r}_{\alpha-1}$ & $0 . \mathrm{a} 41 \mathrm{a} 42 \mathrm{a} 43 \mathbf{a} 44 \ldots \mathrm{a} 4 \mathrm{rr}_{\mathrm{i}}$ \\
\hline & $\ldots$ & $\ldots$ \\
\hline \multirow[t]{4}{*}{ Renglón $\mathbf{r}_{\boldsymbol{\alpha}}$} & $0 . \operatorname{ar}_{\alpha 1} \operatorname{ar}_{\alpha} 2 \operatorname{ar}_{\alpha} 3 \operatorname{ar}_{\alpha} 4 \ldots \operatorname{ar}_{\alpha} r_{\alpha-1}$ & $0 . \operatorname{ar}_{\alpha} 1 \operatorname{ar}_{\alpha} 2 \operatorname{ar}_{\alpha} 3 \ldots \operatorname{ar}_{\alpha} \mathbf{r}_{\alpha} \ldots \operatorname{ar}_{\alpha} r_{i}$ \\
\hline & \multirow{3}{*}{$\begin{array}{l}\text { Ya no pueden existir en el domi- } \\
\text { nio más } r_{\alpha} \in \mathrm{R}_{\alpha} \text { que enumeren a } \\
\text { los posibles reales subsecuentes } \\
\text { en el contradominio universal Rii. }\end{array}$} & \\
\hline & & $0 . \operatorname{ar}_{11} \operatorname{ar}_{1} 2 \operatorname{ari}_{3} \ldots$ arira $+x \ldots$ ariri \\
\hline & & 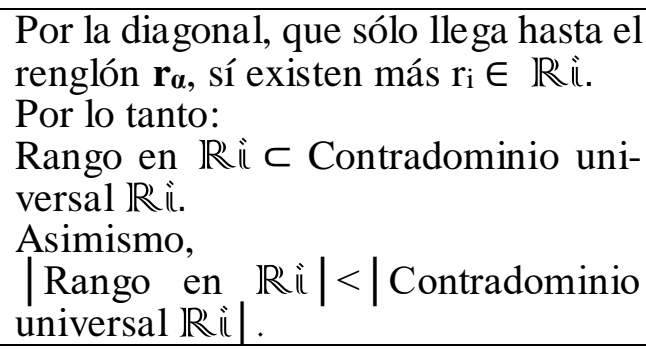 \\
\hline
\end{tabular}

Donde $r_{\alpha-1} \in R_{\alpha-1}, r_{\alpha} \in R_{\alpha}, r_{i} \in \mathbb{R}$ i.. $Y r_{i} \geq r_{\alpha}>r_{\alpha-1}$.

TABLA 4. f:R $\rightarrow$ RRii.

No hemos ido más allá de $\mathbb{R} i i$ o de $\mathbb{R}$. Cualquier número correspondiente al diagonal cognado sobre un $R_{\alpha}$, o a su antidiagonal, es un número real $r_{i} \in \mathbb{R} i i$ o $r \in R$ :

Teorema 12. Cualquier número $r_{\alpha} \in R_{\alpha}$ es, y tiene que ser, un número real:

$\forall \alpha \mathrm{r}_{\alpha} \in \mathrm{R}_{\alpha} \rightarrow \mathrm{r}_{\alpha} \in \mathbb{R}$ ï.

Demostración: Ello ocurre:

1) $O$ bien porque los $r_{1} \in R_{1} \subset R_{\alpha}$ son números reales (por el Lema 2), obtenidos a partir de la primera diagonal cognada;

2) $\mathrm{O}$ bien por ser obtenidos, $\forall \alpha$, los $r_{i} \in R_{\alpha}$ necesariamente a partir de una diagonal de Cantor $\delta$-ampliada, cognada, comenzando por la diagonal sobre $\mathrm{R}_{1}$. En cada una de las cuales diagonales $\delta$-ampliadas se toma (por definición del procedimiento diagonal) una 
cifra $r_{i} \in \mathbb{R}^{\mathrm{i}}$ - por ejemplo, de 0 a 9 en el sistema decimal - de cada número real en el rango. Estando formado el número obtenido, por lo tanto, solamente de dígitos reales. Siendo por ende ese número diagonal un número real que genera en cada instancia un antidiagonal cognado, el cual por definición consta únicamente de los dígitos 1 y 2.

$\mathrm{r}_{\mathrm{i}}$ es un número real por no ser obtenido de un elemento diagonal ajeno. Hecho lógicamente equivalente al que le permitió concluir a Cantor que $|\mathbb{R} i i|>|\mathbb{N}|$. Pues de lo contrario, con un diagonal ajeno, por definición, se obtendría un número $\mathrm{x}$ tal que no garantizaría lógicamente que $\mathrm{x} \in \mathbb{R}$ ii y por lo tanto no se estaría demostrando que $\mathrm{x}$ fuera algún número real que pudiera contribuir a incrementar la cantidad de números de $\mathbb{R} i i$.

En la posibilidad de demostrar que existen los números transfinitos subyace la necesidad de obtener diagonales cognados. Los cuales llevan a la necesidad lógica de la no existencia de un último $R_{\alpha} \subset \mathbb{R}$ y por lo tanto, de la no existencia de un último $r \in \mathbb{R}$.

La respuesta a la pregunta: $¿ \mathbb{R} i \dot{i}=\mathrm{R}\left\lceil_{\aleph_{\alpha}}\right.$ ? es que $\forall \alpha \mathbb{R} i \neq \mathrm{R} \uparrow_{\aleph_{\alpha}}$. Y existen los cardinales $\left|R_{\alpha}\right|=\aleph_{\alpha} y\left|R_{\alpha+1}\right|=\aleph_{\alpha+1}$. Y existen siempre números reales con más que cualquier cantidad $\aleph_{\alpha}$ de cifras. $Y$ así hemos verificado, como anunciamos al comienzo del inciso 3.2, su existencia de manera matemática.

Corolario 5. En la colección de los subconjuntos de la clase $\mathbb{R}$ i i se cumple una propiedad análoga al orden arquimediano de los números naturales: Sea $\left|R_{\alpha}\right|$ cualquier número cardinal transfinito $\aleph_{\alpha}$, entonces existe siempre un $\left|R_{\alpha+1}\right|=\aleph_{\alpha+1}$ mayor que $\left|R_{\alpha}\right|$.

En la Figura 1 se presenta un esquema que muestra gráficamente el sentido de nuestra argumentación, demarcando aquella parte improcedente de inicio en el cálculo de Cantor.

\section{RENORMALIZACIÓN GENERALIZADA}

Con base en lo ya tratado procederemos en seguida a redimensionar $\mathrm{R}_{\alpha}, \mathrm{P}_{\alpha}$ y $\mathbb{R}^{\mathrm{i}} \mathrm{i}$. Con $P_{\alpha}$ $\equiv \alpha$-ésimo conjunto potencia desde $\mathbb{N}$.

Primero vamos a demostrar que $\left|P_{\alpha}\right|=\left|R_{\alpha}\right|$.

Lema 4. Sea $P_{\alpha}$ un conjunto cualquiera en la sucesión de conjuntos potencia a partir de $\mathbb{N}$. Y sea $\mathrm{CPs}$ la cantidad de elementos que pertenecen a cada conjunto $P_{s} \subseteq P_{\alpha_{0}}$ Entonces $\left|C_{P s}\right| \leq \aleph_{\alpha-1}$.

Demostración. $\mathrm{P}_{\alpha}$ tiene a lo más $\aleph_{\alpha-1}$ elementos, por definición de la cardinalidad de $\mathrm{P}_{\alpha}$. Por lo tanto, cada subconjunto $\mathrm{P}_{\mathrm{s}}$ de $\mathrm{P}_{\alpha}$ tiene (por el Axioma Esquema de Separación) a lo más $\aleph_{\alpha-1}$ elementos: $\left|\mathrm{CPs}_{P s}\right| \leq \aleph_{\alpha-1}$. 


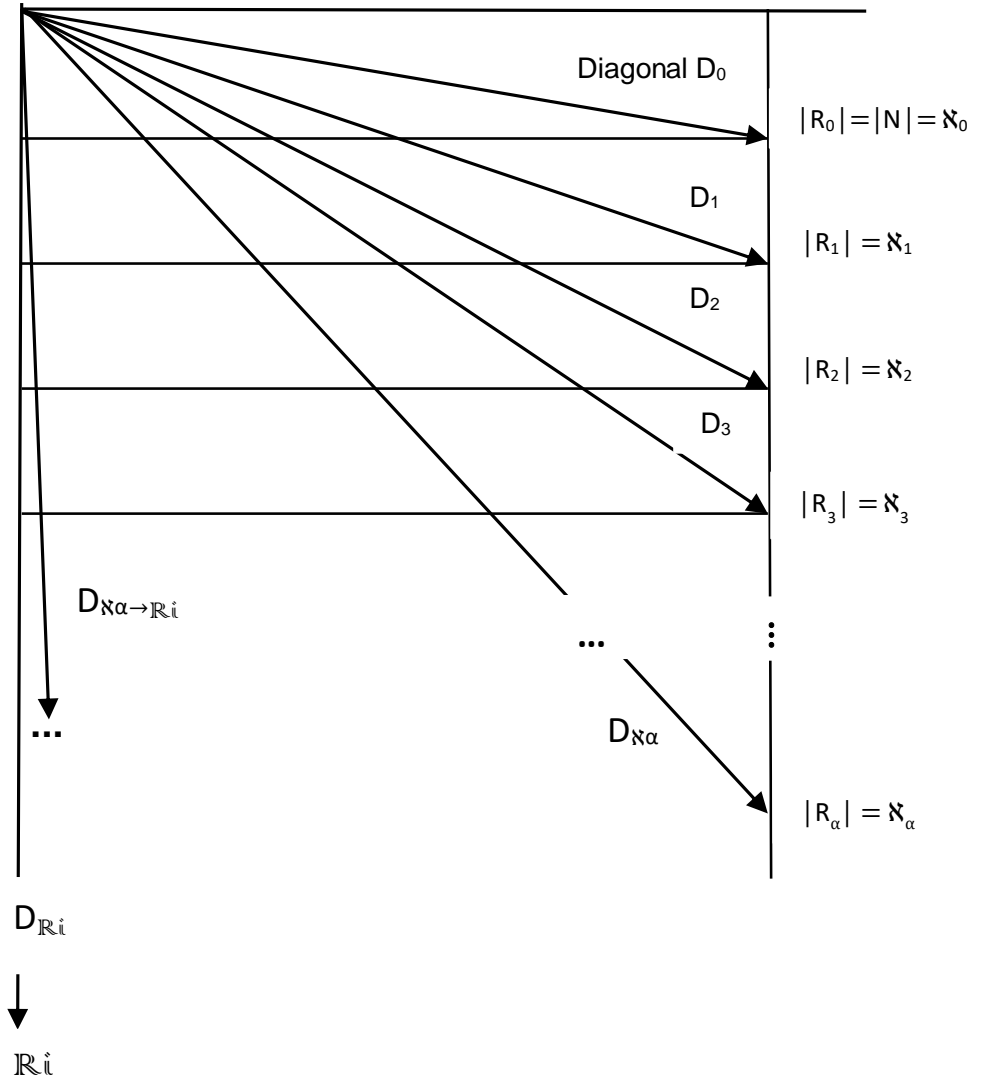

El proceso primigenio de la diagonal de Cantor lo que en realidad traza es la Diagonal Do sobre un conjunto de números reales $\mathrm{R}_{0}$ con una cardinalidad igual a $|\mathbb{N}|$. Pero ello se ha confundido con el trazo y operación de una diagonal $\mathrm{D}_{\mathbb{R i}}$ encima del eje $\mathbb{R}$ ii, creyendo haber abarcado a toda la clase $\mathbb{R} i i$ de un sólo golpe. Cuando lo más que se puede hacer con fundamento lógico es ir construyendo paso a paso los subconjuntos $\mathrm{R}_{\alpha}$ de $\mathbb{R}$ ii, utilizando también las diagonales $\mathrm{D}_{1}, \mathrm{D}_{2}, \ldots, \mathrm{D}_{\boldsymbol{N}_{\alpha}}$, de tal manera que $\mathrm{D}_{\boldsymbol{\aleph}_{\alpha}}$ tiende a coincidir cada vez más con el eje $\mathbb{R} i i$, pero nunca lo alcanza: $\mathrm{D}_{\alpha} \rightarrow \mathbb{R}_{i}$. Matemáticamente no se puede trazar una diagonal $\mathrm{D}_{\mathbb{R} i}$. $Y$, como se puede apreciar a la derecha de la gráfica, cada vez se va ampliando en cardinalidad la cantidad de números reales sobre los que se puede ir operando una diagonal.

FIGURA 1.

Teorema 13. $\forall \alpha\left|R_{\alpha}\right|=\left|P_{\alpha}\right|$. Es decir, $\left|P \uparrow_{\aleph_{\alpha-1}}\right|=\left|R \uparrow_{\aleph_{\alpha-1}}\right|$

Demostración. Usaremos el teorema de Schroeder-Bernstein para demostrar la igualdad:

1. Demostramos primero $\forall \alpha\left|\mathrm{R}_{\alpha}\right| \leq\left|\mathrm{P}_{\alpha}\right|$. O sea, $\forall \alpha\left|\mathrm{R}_{\mathrm{N}_{\alpha-1}}\right| \leq\left|\mathrm{P} \uparrow_{\mathrm{N}_{\alpha-1}}\right|$.

Se establece una función $\mathrm{f}: \mathrm{R}_{\alpha} \rightarrow \mathrm{P}_{\alpha}$, donde es inyectado cada $\mathrm{r}_{\alpha} \in \mathrm{R}_{\alpha}\left(\equiv \mathrm{R} \uparrow_{\boldsymbol{N}_{\alpha-1}}\right)$ hacia cada $\mathrm{P}_{\mathrm{s}} \in \mathrm{P}_{\alpha}$, con $\mathrm{P}_{\mathrm{s}} \subseteq \mathrm{P}_{\alpha-1}$.

Se escribe la parte decimal de cada $r_{\alpha} \in \mathrm{R} \uparrow_{\aleph_{\alpha-1}}$ en forma binaria, como una alternancia de los dígitos 0 y 1 . Decimal que, por definición en el teorema 9, sólo puede tener una cardinalidad de cifras menor o igual a $\aleph_{\alpha-1}:\left|\mathrm{Cr}_{\alpha}\right| \leq \aleph_{\alpha-1}$. 
Establecemos el $\alpha$-ésimo número 1 de la alternancia indicando que el número real $\mathrm{r}_{\alpha} \in$ $\mathrm{P}_{\mathrm{s}}$, mientras que el $\alpha$-ésimo dígito 0 indica que $\mathrm{r}_{\alpha} \notin \mathrm{P}_{\mathrm{s}}$.

Anticipadamente, en la Teoría de Conjuntos se sabe que para evitar repeticiones, en caso de generar el mismo número binario en más de un modo, tomamos sólo aquella forma decimal que no termine únicamente en ceros. Y que, aunque $(0,1)$ no incluya a 0 , que se corresponde con el conjunto vacío, $|(0,1)|=|[0,1)|$.

Por ello cada $\mathrm{r}_{\alpha} \in \mathrm{R}_{\alpha}$ corresponde a un diferente subconjunto $\mathrm{P}_{\mathrm{s}}$ de $\mathrm{P}_{\alpha}$, al ocurrir que $\left|C_{P s}\right| \leq \aleph_{\alpha-1}$ (en virtud del Lema 4) y $\left|C_{\alpha}\right| \leq \aleph_{\alpha-1}$ (por la definición en el Teorema 9), lo cual implica que $\forall \mathrm{P}_{\mathrm{s}} \exists \mathrm{r}_{\alpha}$ tal que $\left|\mathrm{CPs}_{\mathrm{Ps}}\right|=\left|\mathrm{Cr}_{\alpha}\right|$. Y por lo tanto, cualquier $\mathrm{P}_{\mathrm{s}}$ puede ser correspondido en su cantidad de elementos por un $r_{\alpha}$ con semejante cantidad de cifras. Estableciéndose así la inyección de $R_{\alpha}$ en $P_{\alpha}$. De modo que $\left|R_{\alpha}\right| \leq\left|P_{\alpha}\right|$.

2. Se demuestra $\left|P_{\alpha}\right| \leq\left|R_{\alpha}\right|$. O sea, $\forall \alpha\left|P_{\kappa_{\alpha-1}}\right| \leq\left|R_{\aleph_{\alpha-1}}\right|$.

Cada subconjunto de $\mathrm{P}_{\alpha}$ corresponde, como se demostró anteriormente, a una infinita alternancia binaria de números 0 y 1 , de manera que se puede inyectar tal alternancia al $\mathrm{r}_{\alpha}$ número decimal respectivo.

De esta forma, cada subconjunto $\mathrm{P}_{\mathrm{s}} \in \mathrm{P}_{\alpha}$ corresponde a un diferente decimal $\mathrm{r}_{\alpha} \in \mathrm{R}_{\alpha}$ compuesto únicamente de las cifras 0 y 1 , con $\left|\mathrm{Cr}_{\alpha}\right| \leq \mathcal{N}_{\alpha-1}$. Entonces, tenemos $\left|\mathrm{P}_{\alpha}\right| \leq$ $\left|R_{\alpha}\right|$.

Por lo tanto, por 1 y 2 en esta demostración y el Teorema de Schroeder-Bernstein, $\left|P_{\alpha}\right|=\left|R_{\alpha}\right|$,

es decir, existen exactamente tantos números reales en cada $\mathrm{R}_{\alpha}$ como subconjuntos de los reales en cada $\mathrm{P}_{\alpha}$.

Teorema 14. $\left|P_{2}\right|>\left|R_{1}\right|$.

Demostración.

$\left|\mathrm{P}_{2}\right|>\left|\mathrm{P}_{1}\right|$ (por el Teorema de Cantor).

$\left|P_{1}\right|=\left|R_{1}\right|$ (por sustitución en el Teorema 13).

$\left|\mathrm{P}_{2}\right|>\left|\mathrm{R}_{1}\right|$ (por transitividad).

Teorema 15. $\left|R_{2}\right|>\left|P_{1}\right|$.

Demostración.

$\left|\mathrm{R}_{2}\right|>\left|\mathrm{R}_{1}\right|$ (por el Corolario 4).

$\left|R_{1}\right|=\left|P_{1}\right|$ (por el Teorema 6).

$\left|\mathrm{R}_{2}\right|>\left|\mathrm{P}_{1}\right|$ (por transitividad).

Teorema 16. $\forall \alpha\left|R_{\alpha}\right|>\left|P_{\alpha-1}\right|$.

Demostración.

$\left|R_{\alpha}\right|>\left|R_{\alpha-1}\right|$ (por el Teorema 9).

$\left|R_{\alpha-1}\right|=\left|P_{\alpha-1}\right|$ (por sustitución en el Teorema 13).

$\left|\mathrm{R}_{\alpha}\right|>\left|\mathrm{P}_{\alpha-1}\right|$ (por transitividad). 
Se puede apreciar, entonces, que Cantor y la matemática conjuntista después de él, dieron el paso injustificado de identificar al que hemos llamado $\mathrm{R}_{1}$, el único conjunto garantizado lógicamente por el proceso de la diagonal a partir del razonamiento original de Cantor, con $\mathbb{R}$ i y con $\mathbb{R}$. Y por ello también creyeron que comenzando con $\aleph_{2}$, o cualquier cardinal transfinito que $\boldsymbol{\aleph}_{\alpha}$-enumere al conjunto potencia $\mathrm{P}(\mathrm{P}(\mathbb{N})) \equiv \mathrm{P}_{2}$, se rebasa al conjunto $\mathbb{R}$ de todos los números reales. Cuando, como he demostrado, no es así. $\mathrm{P}_{2}$, al rebasar a $\mathrm{P}(\mathbb{N}) \equiv \mathrm{P}_{1}$ a lo que rebasa es a $\mathrm{R}_{1}$. Pero es igual a $\mathrm{R}_{2}$ y menor a $\mathbb{R}$ ii. $\mathrm{Y} \mathrm{P}_{\alpha}$ a lo que rebasa es a $R_{\alpha-1}$, y es igual a $R_{\alpha}$. No se ha ido más allá de $\mathbb{R}^{\prime}$ ï ni de $\mathbb{R}$.

Aquí ya no era obvio, al menos antes de estas demostraciones, lo que sí lo era en el Lema 2 , pues es contrario a la creencia actual en la matemática de que $\aleph_{2}$, en tanto cardinalidad del conjunto potencia $\mathrm{P}\left(\mathrm{P}(\mathbb{N})\right.$ ) (o cualquier otro cardinal transfinito que pudiera $\boldsymbol{\aleph}_{\alpha}$-enumerarlo), rebasa a la cardinalidad de la clase $\mathbb{R}$ de todos los números reales, y que los miembros de $\mathrm{P}(\mathrm{P}(\mathbb{N}))$ son más que los $r \in \mathbb{R}$.

Teorema 17. $\forall \alpha\left|\mathbb{R}^{\prime} i\right|>\left|P_{\alpha}\right|$.

Demostración. Como $\left|\mathrm{P}_{\alpha}\right| \equiv\left|\mathrm{P} \uparrow_{\aleph_{\alpha-1}}\right|$, la cantidad de elementos de cada $\mathrm{P}_{\mathrm{s}} \in \mathrm{P}_{\alpha}$ está limitada por la cardinalidad $\aleph_{\alpha-1}$ (por el Lema 4), mientras que $\mathbb{R}^{\prime} i i$ contiene, además de los ri que corresponden a esos elementos (alcanzando los dígitos de estos ri una cardinalidad $\left|\mathrm{R}_{\alpha-1}\right|$, por el Teorema 16), a aquellos otros $\mathrm{r}_{i}$ que poseen más de $\aleph_{\alpha-1}$ cifras (por el Teorema 11) y que, por lo tanto, forman conjuntos con cardinalidades mayores.

\section{LA HIPÓTESIS DEL CONTINUO}

Cantor, y con él los teóricos que lo han sucedido, al no darse cuenta de los detalles matemáticos y lógicos que aquí se han destacado, han confundido $R_{1} \subset \mathbb{R}^{2} i i$ con $\mathbb{R} i i$ mismo. Y por lo tanto también han confundido a $\left|R_{1}\right|<|\mathbb{R} i|$ con $|\mathbb{R} i i|$.

Como he demostrado, para ser lógicamente consistentes con las premisas y los argumentos del proceso diagonal de Cantor, sería un error pensar que a partir de $\mathrm{R}_{1}$ cada diagonal da lugar a una cantidad transfinita nueva que no puede ser $\aleph_{\alpha}$-enumerada con los números reales (con lo que se formaría así una cantidad de elementos adicionales como, por ejemplo, conjuntos de conjuntos, que ya no sería $\aleph_{\alpha}$-enumerable por el conjunto $\mathbb{R}$ ii). Y que así tendríamos $\left|\mathbb{R}^{2} i\right|=|\mathbb{R}|=|\mathrm{P}(\mathbb{N})|$, y $|\mathrm{P}(\mathrm{P}(\mathbb{N}))|>|\mathbb{R}|$.

Cuando en realidad $\left|\mathrm{P}_{1}\right|=\left|\mathrm{R}_{1}\right|$ y $\left|\mathrm{P}_{2}\right|=\left|\mathrm{R}_{2}\right|$. $\mathrm{Y}|\mathbb{R}|>|\mathrm{P}(\mathrm{P}(\mathbb{N}))|$.

Queremos enfatizar y dejar claramente establecido que aquí llamamos $\left|\mathrm{R}_{1}\right|$ y por lo tanto $\aleph_{1}$, o sea $|\mathrm{P}(\mathbb{N})|$, al primer $\aleph$ que es posible obtener a partir de la operación diagonal de Cantor en la función $\mathrm{f}: \mathbb{N} \rightarrow \mathbb{R}$ ii. Con lo cual, con este criterio, si se demostrase la existencia de un $\aleph$ entre $\aleph_{0}$ y $\aleph_{1}$, a tal $\aleph$ se le podría asignar, por ejemplo, un subíndice fraccionado o decimal $\left(\aleph_{1 / 2}\right.$ ó $\aleph_{0.1}$, etc. $)$.

Se llama el continuo $\mathfrak{c}$ a la cardinalidad de $\mathbb{R}$, lo cual relaciona los números reales (el continuo aritmético) con los puntos en una línea (el continuo geométrico). Hablando con lógica estricta, de los teoremas 3,11 y 17 se deduce el siguiente teorema (siendo así consistentes con los resultados hallados hasta aquí a partir de las aserciones matemáticas asumidas en el proceso de la diagonal de Cantor, de las cuales proceden también nuestras ampliaciones):

Teorema 18: Asúmase que el método diagonal de Cantor es consistente. Entonces 
Es decir, en el contexto de las premisas y argumentos matemáticos y lógicos propios del proceso de la diagonal, es falso que si $R_{\alpha} \subset \mathbb{R}$ es un conjunto no enumerable por $\mathbb{N}$, entonces existe una biyección $f: R_{\alpha} \rightarrow \mathbb{R}$. Esto es, es falso que

$\forall \alpha R_{\alpha} \subset \mathbb{R} \wedge\left|R_{\alpha}\right|>|\mathbb{N}| \leftrightarrow\left|R_{\alpha}\right|=|\mathbb{R} i|$

O sea, no es verdadero que

$\forall \alpha \mathrm{R}_{\alpha} \subset \mathbb{R} \rightarrow \aleph_{\alpha}>\aleph_{0} \leftrightarrow \aleph_{\alpha}=|\mathbb{R} i|$.

Demostración. Si suponemos que el procedimiento de Cantor de la diagonal es correcto, entonces, por los teoremas 3, 11 y 17, derivados a partir de aceptar esa validez (de la cual provienen sus ampliaciones en este escrito), no puede existir ninguna biyección f: $R_{\alpha} \rightarrow$ $\mathbb{R}$. Por lo tanto, cualquier $\mathrm{R}_{\alpha}$ es un subconjunto propio de $\mathbb{R}$ y con cardinalidad no igual a él:

$\forall \alpha R_{\alpha} \subset \mathbb{R} \wedge\left|R_{\alpha}\right|<|\mathbb{R}|$

Y por lo tanto, ningún $\aleph_{\alpha}>\aleph_{0}$ es igual a $|\mathbb{R} i i|$ :

$\forall \alpha \neg \exists \aleph_{\alpha} \geq|\mathbb{R} i|$.

Así tenemos que

$|\mathbb{N}|=\left|\mathrm{R}_{0}\right|<\left|\mathrm{R}_{1}\right|<\left|\mathrm{R}_{2}\right|<\left|\mathrm{R}_{3}\right|<\ldots\left|\mathrm{R}_{\alpha}\right| \ldots<|\mathbb{R}|$

Por lo tanto entre $\mathbb{N}$ y el continuo $c=|\mathbb{R}|$ hay una infinita cantidad de conjuntos infinitos determinados, $\mathrm{R}_{\alpha}$, con cardinalidades distintas entre sí. Cada uno de ellos formado por números reales.

Por otra parte, cualquier conjunto infinito determinado $\mathrm{P}_{\alpha}$, o su equivalente en cardinalidad, puede ser $\aleph_{\alpha}$-numerado, medido, por un subconjunto $R_{\alpha}$ de $\mathbb{R}$, por el Teorema 17.

Asimismo, no existe algún número transfinito $\aleph_{\alpha}$ que sea mayor a la cardinalidad de $\mathbb{R}$ (por los teoremas 11 y 13). Consecuentemente, no existe el problema de si entre la cardinalidad de $\mathbb{R}$ y la cardinalidad $\aleph_{\alpha}$ de un conjunto mayor a $\mathbb{R}$ hay un $N$ intermedio. $O$ si entre conjuntos de (imposible) cardinalidad $\aleph_{\alpha}$ mayores a $\mathbb{R}$ pueda haber $\aleph$ intermedios.

La Hipótesis del Continuo, como está formulada en la actualidad, es errónea no sólo porque la deducción rigurosa niegue que

Todos los conjuntos infinitos de puntos $\mathrm{P}$ tienen ya sea la potencia de la primera clase numérica (I) o la potencia de la segunda clase numérica (II) [viz. la potencia de los números naturales o la del continuo. Nota del autor]. (Cantor [2], p. 905)

Sino también porque, por los teoremas 3, 11 y 17, entre ambas variedades lineales infinitas se encuentran no una, sino infinitas variedades $\aleph_{\alpha}$. El continuo, el cuerpo de los números reales, o cualquier intervalo del mismo, como $(0,1)$, está infinitamente alejado de $\aleph_{1}$ o de cualquier $\aleph_{\alpha}$ en cuanto a cardinalidad.

Corolario 6. Como se ve, a partir de la operación diagonal, $\mathrm{c} \neq \aleph_{1}$, pues $\mathfrak{c}=|\mathbb{R}|>\aleph_{1}$.

Y, además:

Teorema 19. Asúmase que el método diagonal de Cantor es consistente. Entonces 
$2^{\mathrm{K}_{0}}$ no es la cardinalidad del continuo:

$\mathfrak{c} \neq 2^{\mathrm{K}_{0}}$

$c>2^{\aleph_{0}}$.

Demostración. $2^{\aleph_{0}}$ es un infinito determinado, como se establece a partir del Teorema de Cantor acerca de los conjuntos potencia. Y le corresponde por ello un cardinal $\aleph_{\alpha}$, al menos el $\aleph_{1}$. Pero como ya se ha demostrado aquí con los teoremas 11 y 17 , todo $\aleph_{\alpha}$ es menor que $|\mathbb{R}|$.

Por lo tanto $2^{\aleph_{0}}$ es menor que $|\mathbb{R}|$ :

$2^{\aleph_{0}} \neq|\mathbb{R}| ;|\mathbb{R}|>2^{\aleph_{0}}$.

$\mathrm{Y}$ como $|\mathbb{R}|=\mathfrak{c}$, entonces $2^{\boldsymbol{K}_{0}}$ es menor que $\boldsymbol{c}$.

Teorema 20. En tanto primera aplicación del método de la diagonal en $f: \mathbb{N} \rightarrow \mathbb{R}$ ï, $2^{\boldsymbol{N}_{0}}$ es la cardinalidad de $R_{1}$, que es igual a $\aleph_{1}$ (es decir al número transfinito que sigue a $\aleph_{0}$ cuando se aplica el proceso de la diagonal):

$2^{\aleph_{0}}=\left|R_{l}\right|$.

Demostración. Por derivación del Teorema de Cantor en la Teoría de Conjuntos, $2^{\mathrm{N}_{0}}=$ $\left|\mathrm{P}_{1}\right|=\aleph_{1}$.

Por el Teorema 13, $\left|\mathrm{P}_{1}\right|=\left|\mathrm{R}_{1}\right|=\mathrm{N}_{1}$.

Por reemplazo de $\left|\mathrm{P}_{1}\right|$ por $\left|\mathrm{R}_{1}\right|, 2^{\mathrm{N}_{0}}=\left|\mathrm{R}_{1}\right|=\aleph_{1}$.

A partir, acentúo, de las premisas del proceso de la diagonal de Cantor, el continuo no es ni puede ser igual a $\aleph_{2}$, ni a $\aleph_{3}$, ni a $\aleph_{26}$, ni a $\aleph_{\omega}$, ni a cualquier otro $\aleph_{\alpha}$. Y de ninguna manera $2^{\mathrm{N}_{0}}$ puede ser mayor que todos los otros $\left|\mathrm{R}_{\alpha}\right|>\left|\mathrm{R}_{1}\right|$.

Precisamente, esta refutación de la Hipótesis del Continuo tiene la ventaja de permitirnos ver rigurosamente el alcance del cuerpo de los números reales, frente a meras conjeturas intuitivas como las de Baire, Gödel o Cohen (cf. Dauben [6], p. 269) respecto a la riqueza del mismo continuo.

Con el método cantoriano de la diagonal consecuentemente aplicado no hay forma de rebasar con los $\aleph_{\alpha}$, cardinalmente, a $\mathbb{R}$, en virtud del carácter del antidiagonal cognado necesario para su argumentación, el cual siempre resulta ser un número real.

\section{UNA NUEVA HIPÓTESIS: LA CONTINUIDAD DENTRO DEL CONTINUO}

Pero surgen dos hipótesis, parecidas en algo pero en realidad muy diferentes a la HC (que por sus características llamaremos las Hipótesis de la Continuidad al Interior del Continuo o HCIC).

1. La más limitada: entre $|\mathrm{N}|\left(=\left|\mathrm{R}_{0}\right|\right)$ y $\left|\mathrm{R}_{1}\right|$, ambas menores a $|\mathbb{R}|$, no existe una cardinalidad intermedia (por ejemplo $\left|\mathrm{R}_{0.5}\right|$ ) a aquella obtenida mediante la operación de la diagonal. O lo que es lo mismo, entre $\aleph_{0}$ y $\aleph_{1}$ no existe un $\aleph$ intermedio.

Es decir:

HIPÓTESIS RESTRINGIDA DE LA CONTINUIDAD AL INTERIOR DEL CONTINUO(O: HIPÓTESIS RESTRINGIDA DE LA CONTINUIDAD ENTRE $\left.\aleph_{0} Y 2^{\aleph_{0}}\right)$ : 
Si $R_{\beta} \subset R_{l}$ es un conjunto no enumerable por $\mathbb{N}$, entonces existe una biyección $f: R_{\beta} \rightarrow$ R. Esto es, $\left|\mathrm{R}_{\beta}\right|>|\mathbb{N}| \leftrightarrow\left|\mathrm{R}_{\beta}\right|=\left|\mathrm{R}_{1}\right|$.

Entonces, para cualquier operación matemática posible, además de ser válido para la operación diagonal de Cantor, $\aleph_{1}=2^{\aleph_{0}}=\left|R_{1}\right|=\left|P_{1}\right|(\neq|\mathbb{R}|)$.

En este replanteamiento de ninguna manera puede $\aleph_{1}$, ni $2^{\aleph_{0}}, \aleph_{\alpha}$-enumerar a todos los reales en $\mathbb{R}$. Pues ambas $\aleph_{\alpha}$-enumeran solamente a $R_{1}$. La pregunta sigue siendo si entre $\aleph_{0}$ y $2^{\aleph_{0}}$ existe otro cardinal; pero ya no es equivalente a si entre $\aleph_{0}$ y el continuo $c$ existe un cardinal intermedio.

2. La Hipótesis más general: entre cada $\left|R_{\alpha}\right|$ y $\left|R_{\alpha+1}\right|$ no existe una cardinalidad intermedia. O lo que es lo mismo, entre cada $\aleph_{\alpha}$ y $\aleph_{\alpha+1}$ no existe un $\aleph$ intermedio. Es decir:

HIPÓTESIS GENERALIZADA DE LA CONTINUIDAD AL INTERIOR DEL CONTINUO (o: HIPÓTESIS GENERALIZADA DE LA CONTINUIDAD ENTRE $\aleph_{\alpha} Y 2^{\left.N_{\alpha}\right):}$

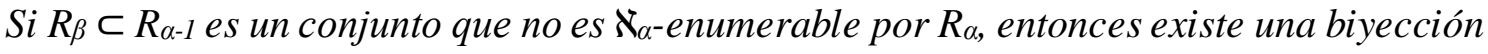

$f: R_{\beta} \rightarrow R_{\alpha+1}$. Esto es, $\left|\mathrm{R}_{\beta}\right|>\left|\mathrm{R}_{\alpha}\right| \leftrightarrow\left|\mathrm{R}_{\beta}\right|=\left|\mathrm{R}_{\alpha+1}\right|$.

Así tendríamos, para cualquier posible operación matemática, además de ser válido para la operación diagonal de Cantor:

$\forall \alpha \aleph_{\alpha+1}=2^{\aleph_{\alpha}}=2^{\mid \mathrm{R} \alpha}\left|=2^{\mid \mathrm{P} \alpha}\right|=\left|\mathrm{P}_{\alpha+1}\right|=\left|\mathrm{R}_{\alpha+1}\right|(\neq|\mathbb{R}|)$.

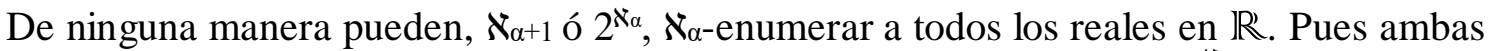
$\aleph_{\alpha}$-enumeran solamente a $R_{\alpha+1}$. La pregunta sigue siendo si entre $\aleph_{\alpha}$ y $2^{\aleph_{\alpha}}$ (ambos dentro del continuo y menores en cardinalidad a él), existe otro cardinal.

Pero ya no es equivalente a si entre un $\aleph_{\alpha}$ interior al continuo y el continuo $c$ existe un cardinal intermedio. $\mathrm{O}$ si entre cualquier $\aleph_{\alpha}$ igual, o mayor y exterior, al continuo (un $\aleph_{\alpha}$ que ahora sabemos inexistente si el método de la diagonal de Cantor es consistente) y otro $\aleph_{\alpha+1}$ (también exterior y mayor al continuo; y del mismo modo inexistente) hay un cardinal intermedio. Puesto que $2^{\mathrm{Na}_{\alpha}}$ no es la cardinalidad del continuo.

Ya en ninguna de esas hipótesis interviene el continuo como la totalidad del conjunto de puntos de una recta numérica, en tanto que, como ha sido demostrado en este artículo, ningún subconjunto de puntos $\mathbb{R}_{\alpha}$ es el continuo $\mathfrak{c}$, o $\mathbb{R}$ ii o $\mathbb{R}$, sino un subconjunto propio transfinito de menor tamaño del mismo.

Entonces, probamos que dentro del continuo, entre $\mathbb{N}$ y el continuo, existen infinitos conjuntos $\mathrm{R}_{\alpha}$, tanto numéricos como de puntos, con distintas cardinalidades $\aleph_{\alpha}$. Y que espera respuesta la cuestión de si entre esos conjuntos $\mathrm{R}_{\alpha}$ (o sus equivalentes $\mathrm{P}_{\alpha}$ ) existen a su vez otros conjuntos intermedios con cardinalidades diferentes a tales $\aleph_{\alpha}$.

Teorema 21. Asumase que la Hipótesis del Continuo HC $(\neq H C I C)$ es verdadera, entonces el método de la diagonal de Cantor es inconsistente.

Demostración. Por Modus Tollens aplicado al Teorema 18.

Pero lo que afecta a la HC no afecta a la HCIC. Respecto a ésta (o a la $\neg$ HCIC) sí hace falta probar si es verdadera o falsa, o consistente o inconsistente en relación al método de la diagonal o bien a diferentes axiomas.

Entonces, al no considerar Cantor y sus continuadores las consecuencias estrictamente lógicas de su argumento, a partir del papel que juega la cantidad de cifras de cada diagonal 
cognada, que demuestro en este artículo, han concebido la Hipótesis del Continuo de manera en alguna medida errónea.

En tal dirección Gödel afirmaba:

(1)

El problema del continuo de Cantor no es más que la pregunta: ¿Cuántos puntos hay en una línea recta de un espacio euclídeo?

(2)

Una pregunta equivalente es: ¿Cuántos conjuntos diferentes de números naturales existen? (Gödel [11], p. 354)

Pero, por lo ya manifestado, es equivocado plantearlo así, diferente a: ¿Cuántos conjuntos diferentes $\mathrm{R}_{\alpha}$ de números reales existen?

En realidad (2) es distinto de (1), y no es (1) dicho en otros términos. Pues los conjuntos de enteros se agotan con $\mathrm{R}_{1}$; les siguen en cardinalidad los conjuntos de números reales $\mathrm{r}_{2} \in \mathrm{R}_{2}$; luego los conjuntos de números reales $\mathrm{r}_{3} \in \mathrm{R}_{3}$, etc. Con $\mathrm{R}_{1} \subset \mathrm{R}_{2}, \mathrm{R}_{2} \subset \mathrm{R}_{3}$, etc. Existen infinitos subconjuntos $\mathrm{R}_{\alpha}$ de reales (de trascendentales) y de puntos en la línea recta (y en sus respectivas relaciones $\alpha$-arias, en el plano o en $\alpha$ dimensiones) cada uno con distinta potencia $\aleph_{\alpha}$.

$2^{\aleph_{0}}$ está localizado en la jerarquía alef, en cuanto derivado de la aplicación de la diagonal, en su segundo peldaño, $\left|\mathrm{R}_{1}\right|$. Y no puede estarlo en nada mayor. Mientras que el continuo, la clase de los puntos sobre una línea recta en el espacio Euclideano, es mucho más rico en elementos.

Por su parte, Cohen sostenía que

Un punto de vista que el autor siente puede llegar a ser aceptado en algún momento es que la $\mathrm{HC}$ es obviamente falsa. La principal razón por la que uno acepta el Axioma de Infinitud es probablemente porque sentimos que es absurdo que el proceso de agregar sólo un conjunto a la vez puede ser exhaustivo con el universo entero. Similarmente con los axiomas de infinitud más elevados. Ahora $\aleph_{1}$ es el conjunto de los ordinales contables y éste es meramente una forma especial y la más simple de generar un cardinal más elevado. El conjunto $\mathrm{C}$ [el continuo; para Cohen igual a un $\mathrm{P}_{\alpha} \mathrm{y}$ a un $2^{\mathrm{N}_{\alpha}}$. Nota del traductor] es, en contraste, generado por un principio totalmente nue vo y más poderoso, es decir el Axioma del Conjunto Potencia. Es irrazonable esperar que cualquier descripción de un cardinal más grande que intente construir tal cardinal a partir de ideas derivadas del Axioma de Reemplazo pueda alguna vez alcanzar C. Así C es mayor que $\aleph_{n}, \aleph_{\omega}, \aleph_{\alpha}$ donde $\alpha=\aleph_{\omega}$ etc. Este punto de vista contempla a C como un conjunto increíblemente rico dado a nosotros por un atre vido axioma nuevo, el cual nunca puede ser aproximado por ningún proceso fragmentario de construcción. (Cohen [5], p.151, traducción propia)

Pero el continuo, como ya vimos, no puede ser generado por el Axioma del Conjunto Potencia, pues es un límite inalcanzable para el proceso de la diagonal, i.e., para la operación $2^{\aleph_{\alpha}}$. El continuo no es representado por $2^{\aleph_{0}}$ o por $\aleph_{2}, \aleph_{15}, \ldots, \aleph_{\alpha}$, como también podía hipotetizar Cohen. $2^{\mathrm{N}_{0}}$ es la cardinalidad de $\mathrm{R}_{1}$. El continuo es algo increíblemente mucho más rico, mucho más suculento, infinitamente más suculento, que $\left|\mathrm{R}_{1}\right|$, i.e. que $\left|P_{1}\right|, \aleph_{1}$ o $2^{\aleph_{0}}$. O que cualquier $\left|R_{\alpha}\right|$, es decir, que $\left|P_{\alpha}\right|, \aleph_{\alpha} O 2^{\aleph_{\alpha}}$.

Lo esencial es que ningún $2^{\boldsymbol{N}_{\alpha}}$ es igual a c. La Hipótesis del Continuo referida a $\mathbf{c}$ se ha demostrado es falsa. Mientras que la hipótesis referida al no continuo $2^{\mathrm{Na}}$ continúa en suspenso. Y es la HCIC. 
En un sentido preciso Gödel y Cohen son inexactos respecto al continuo. Pero debe quedar claro que con ésta refutación y posterior modificación de la Hipótesis del Continuo, la equivocación no ocurre en el desarrollo formal de sus demostraciones de independencia.

Siguen teniendo vigencia lógica las fases de los razonamientos de Gödel y de Cohen, pero hay que corregirlas (como ya corregimos otra demostración de la Teoría de Conjuntos en los teoremas 6 y 13) y aplicarlas a decidir la verdad o falsedad, o la independencia, de una nueva Hipótesis de la Continuidad al Interior del Continuo (de ausencia de cardinalidades intermedias entre los subconjuntos $\aleph_{\alpha}$ del Continuo). Considerando la consistencia de su afirmación (o de su negación $\neg \mathrm{HCIC}$ ), respecto a sistemas como ZFC o afines (y ya no aplicarlas al resuelto dilema $\mathrm{HC}$ o $\neg \mathrm{HC}$ ).

Es decir, las demostraciones de independencia axiomática son válidas siempre y cuando se corrija y evite el error básico de confundir $\mathbb{R}^{\prime i}$ o $\mathbb{R}$ con $R_{1}$ o con cualquier $R_{\alpha}$. $Y$ consiguientemente, haciendo las respectivas sustituciones explícitas o implícitas de $\mathrm{R}_{1} \mathrm{o}$ $\mathrm{R}_{\alpha}$ en vez de $\mathbb{R}_{\mathrm{o}}$ o $\mathbb{R}^{\mathrm{i} i}$ en sus argumentos, modelos y métodos como el Modelo Interno o el Forzado. Y asignando $\aleph_{1}$ sólo a $\mathrm{R}_{1} \mathrm{o}$ a $\mathrm{P}_{1}$, y a sus números ordinales respectivos. $\mathrm{Y}$ cada $\aleph_{\alpha}$ sólo a su pertinente $\mathrm{R}_{\alpha} \mathrm{O} \mathrm{P}_{\alpha}$.

Pero con las demostraciones de Gödel y Cohen adecuadamente rectificadas, se puede probar que tanto HCIC como $\neg \mathrm{HCIC}$ son consistentes con $\mathrm{ZFC}$ o sistemas afines, con el procedimiento de la diagonal y, por lo tanto, con la construcción de los números transfinitos. Todo lo cual lo queremos exponer en detalle en un artículo posterior.

Por otra parte, el Teorema 9 es una demostración lógicamente similar a la del Teorema de Cantor, acerca de la existencia de una sucesión infinita de conjuntos potencia a partir de las respectivas diagonales. Excepto que así tenemos: $|\mathrm{P}(\mathbb{N})| \neq|\mathbb{R} i|$; en cambio, $|\mathrm{P}(\mathbb{N})|=\left|\mathrm{R}_{1}\right| ; \mathrm{y}$ para todo conjunto potencia $\mathrm{P}_{\alpha},\left|\mathrm{P}_{\alpha}\right|=\left|\mathrm{R}_{\alpha}\right|$. Y $\mathbb{R} \sim \mathbb{P}^{\prime}$ (la clase $\mathbb{R}^{2}$ de los números reales es equivalente a la clase de todos los conjuntos potencia). Sobre esto último abundaremos para un siguiente artículo, y además se tratará de los ordinales transfinitos.

La diferencia importante es que la demostración aquí presentada es realizada directamente con diagonales sobre el conjunto de los números reales. Pero solamente así hemos logrado ver algo que no permite apreciar el Teorema de Cantor con referencia a los conjuntos potencia: su real calibración, su medida correcta; y, para el Análisis Matemático, la complejidad y el alcance de los números reales respecto a los conjuntos transfinitos.

\section{REFERENCIAS.}

[1] Cantor Georg. Contributions to the founding of the theory of transfinite numbers. Dover Publications Inc., New York, N. Y., 1952.

[2] Cantor Georg. Foundations of a general theory of manifolds: a mathematico-philosophical investigation into the theory of the infinite. 1883. Ewald William, ed. From Kant to Hilbert: A Source Book in the Foundations of Mathematics. Volume II. Clarendon Press, Oxford University Press, New York, 2005, 881-919.

[3] Cantor Georg. On an elementary question in the theory of manifolds. 1891. Ewald William, ibid., 920-922.

[4] Cohen Paul J. The independence of the Continuum Hypothesis. Proceedings of the National Academy of Sciences of the United States of America, vol. 50 (1963), pp. 11431148, y vol. 51 (1964), pp. 105-110. 
[5] Cohen Paul J. Set Theory and the Continuum Hypothesis. W. A. Benjamin, Inc., New York, 1966.

[6] Dauben Joseph Warren. Georg Cantor: His mathematics and philosophy of the infinite. Princeton University Press, Princeton, NJ, 1990.

[7] Euclides. Elementos. Ed. Gredos. Madrid 1991.

[8] Fraenkel Abraham A. Abstract set Theory. North-Holland Publishing Company, Amsterdam, 1966.

[9] Fraenkel Abraham A. Teoría de los conjuntos y lógica. UNAM, México D.F, 1976.

[10] Gödel Kurt. La consistencia del axioma de elección y de la hipótesis generalizada del continuo con los axiomas de la teoría de conjuntos, 1940. En Obras Completas, Alianza Editorial, S. A. Madrid, 2006.

[11] Gödel Kurt. ¿Qué es el problema del Continuo de Cantor?, 1947, en Obras Completas, Ibid.

[12] Gray, Robert. Georg Cantor and transcendental numbers. The American Mathematical Monthly, vol. 101, November 1994, 819-832.

[13] Jech Thomas. Set Theory. Springer-Verlag, Berlin, 2006.

[14] Suppes Patrick, Teoría Axiomática de Conjuntos. Ed. Norma, Cali, 1968.

[15] Torreti, Roberto. El Paraíso de Cantor. Universidad Nacional Andrés Bello, Santiago de Chile, 1998.

[16] Zermelo Ernst. Investigations in the foundations of set theory I. 1908, Jean van Heijenoort, From Frege to Gödel. Harvard University Press, Cambridge, Massachusetts, 1967, 199-215.

Alejandro Matus. Av. Coyoacán, Col. Del V alle, CD. De México. C.P. 03100. MÉXICO.

E-mail address: alejandromatusunfe020@gmail.com 